\title{
Replacing adenoviral vector HVR1 with a malaria B cell epitope improves immunogenicity and circumvents preexisting immunity to adenovirus in mice
}

\author{
Takayuki Shiratsuchi, ${ }^{1}$ Urvashi Rai, ${ }^{1}$ Anja Krause,2 Stefan Worgall,2 and Moriya Tsuji1 \\ ${ }^{1}$ HIV and Malaria Vaccine Program, Aaron Diamond AIDS Research Center, New York, New York, USA. ²Department of Genetic Medicine, \\ Weill Medical College of Cornell University, New York, New York, USA.
}

\begin{abstract}
Although adenovirus (Ad) has been regarded as an excellent vaccine vector, there are 2 major drawbacks to using this platform: (a) Ad-based vaccines induce a relatively weak humoral response against encoded transgenes, and (b) preexisting immunity to Ad is highly prevalent among the general population. To overcome these obstacles, we constructed an Ad-based malaria vaccine by inserting a B cell epitope derived from a Plasmodium yoelii circumsporozoite (CS) protein (referred to as the PyCS-B epitope) into the capsid proteins of WT/CS-GFP, a recombinant Ad expressing P. yoelii CS protein and GFP as its transgene. Multiple vaccinations with the capsid-modified Ad induced a substantially increased level of protection against subsequent malaria challenge in mice when compared with that of unmodified WT/CS-GFP. Increased protection correlated with augmented antibody responses against the PyCS-B epitope expressed in the capsid. Furthermore, replacement of hypervariable region 1 (HVR1) of the Ad capsid proteins with the PyCS-B epitope circumvented neutralization of the modified Ad by preexisting Ad-specific antibody, both in vivo and in vitro. Importantly, the immunogenicity of the Ad-containing PyCS-B epitope in the HVR1 and a P. yoelii CS transgene was maintained. Overall, this study demonstrates that the HVR1-modifed Ad vastly improves upon Ad as a promising malaria vaccine platform candidate.
\end{abstract}

\section{Introduction}

Malaria remains the world's most salient and prevalent tropical parasitic disease. Each year there are 300-500 million clinical cases diagnosed, with more than 1 million deaths annually; children in sub-Saharan African make up the majority of these cases (1). Despite the vast resources and research aimed at preventing malaria since the 1950s, the fact remains that a malaria vaccine is still urgently needed in order to significantly reduce the overall mortality and morbidity. This, in turn, will remove a major obstacle to the social and economic development of many developing countries.

Immunization with irradiated sporozoites has been previously shown to protect against malaria in not only rodents (2) and monkeys (3) but also in humans $(4,5)$. This clearly demonstrates the feasibility of achieving complete resistance against malaria infection through vaccination. It has been shown that the sporozoite-induced protective immunity is mediated by neutralizing antibodies, which recognize the repeat domain of the circumsporozoite (CS) protein, the major surface antigen of sporozoites (6). The neutralizing effects of anti-repeat antibodies to the CS proteins of Plasmodium falciparum and Plasmodium vivax were demonstrated in chimpanzees (7). In addition to antibodies, $\mathrm{T}$ cells, particularly $\mathrm{CD} 8^{+}$cytotoxic $\mathrm{T}$ cells, are shown to contribute to antimalaria immunity by inhibiting the development of the liver stages of the parasite (8).

Among a variety of recombinant viral vectors (9-12) examined, adenovirus (Ad) has been shown to be an excellent viral vector for a malaria vaccine, due to its ability to induce a potent antigen-spe-

Conflict of interest: The authors have declared that no conflict of interest exists. Citation for this article: J Clin Invest. 2010;120(10):3688-3701. doi:10.1172/JCI39812. cific $\mathrm{CD}^{+} \mathrm{T}$ cell response. However, there are 2 major obstacles that prevent this platform from applying to a malaria vaccine: (a) the inability to induce a potent humoral response against a transgene product, and (b) the widespread preexisting immunity to Ad among the general population, especially Ad serotype 5 (Ad5), which hampers the immunogenicity of any Ad-based vaccine.

Recently, a new approach has been taken in an attempt to augment Ad-induced humoral response by inserting a B epitope in Ad capsid proteins, such as hexon, fiber, penton, and PIX (13-16). Also, in an effort to circumvent preexisting immunity to Ad5, other Ad serotypes with lower seroprevalence, such as Ad11, Ad35, Ad26, Ad48, Ad49, and Ad50, have been evaluated as alternative Ad vaccine platforms. These other Ad serotypes have been shown to induce immune response to a transgene, despite of the presence of anti-Ad5 immunity $(17,18)$. Substitution of Ad5 hexon, which among capsid proteins is the main target of neutralizing antibodies, with that of other serotypes has also been constructed in order to escape preexisting anti-Ad5 immunity $(19,20)$.

For this study, in an attempt to enhance humoral response to the P. yoelii CS protein and circumvent preexisting immunity to Ad5, we have constructed and analyzed what we believe to be several novel recombinant Ads (rAds), which express a B epitope of the P. yoelii CS protein of malaria parasites, in hexon and/or fiber, in addition to containing the P. yoelii CS transgene.

\section{Results}

Construction and in vitro characterization of capsid-modified Ad. The rAds constructed and used in this study are listed in Table 1. The WT/ GFP is a rAd expressing a GFP alone in its transgene, whereas WT/ 
Table 1

rAds constructed and used in this study

$\begin{array}{lcc}\text { rAd } & \text { Capsid } & \text { Transgene } \\ \text { WT/Empty } & \text { WT } & \text { None } \\ \text { WT/GFP } & \text { WT } & \text { GFP } \\ \text { WT/CS-GFP } & \text { WT } & \text { P. yoelii CS and GFP } \\ \text { B-HVR1/CS-GFP } & (\mathrm{QGPGAP})_{3} \text { in HVR1 } & \text { P. yoelii CS and GFP } \\ \text { B-HVR5/CS-GFP } & (\mathrm{QGPGAP})_{3} \text { in HVR5 } & \text { P. yoelii CS and GFP } \\ \text { B-Fib/CS-GFP } & (\mathrm{QGPGAP})_{3} \text { in fiber }(\mathrm{HI} \text { Loop) } & \text { P. yoelii CS and GFP } \\ \text { B-HVR1/B-Fib/CS-GFP } & (\mathrm{QGPGAP})_{3} \text { in HVR1 and fiber } & \text { P. yoelii CS and GFP } \\ \text { WT/P. falciparum CS } & \text { WT } & \text { P. falciparum CS } \\ \text { NANP-HVR1/P. falciparum } \mathrm{CS} & (\mathrm{NANP})_{4} \text { in HVR1 } & \text { P. falciparum CS }\end{array}$

lines stably transfected with coxsackie and Ad receptor (CAR). Although these parental cell lines are hardly infected with Ad, CAR/A20 and CAR/L1210 cells can be infected with Ad in a CAR-dependent manner (data not shown). We found that there was no difference in infectivity to AD293 cells and mouse DCs among rAds. However, fiber-modified Ad showed a slightly lower infectivity to CAR/A20 and CAR/L1210 cells (Figure 4), indicating that the PyCS-B epitope insertion in the HI loop of fiber has an inhibitory effect on the interaction between fiber and CAR, which has been shown as the receptor for fiber (21).

P. yoelii CS-specific immune response and protection induced by capsid-modified Ad in mice. As the rAds had the entire P. yoelii CS protein in the transgene and

CS-GFP is a rAd that expresses P. yoelii CS (GenBank ID J02695.1) and GFP in its transgene. P. yoelii CS protein contains 2 tandem repeats of (QGPGAP) ${ }_{19}$ and (PPQQ $)_{7}$ in the central domain and a $\mathrm{H}-2 \mathrm{~K}^{\mathrm{d}}$-restricted $\mathrm{CD}^{+} \mathrm{T}$ cell epitope, SYVPSAEQI, in the $\mathrm{C}$ terminus. As shown in Table 1 and Figure 1, all capsid-modified Ads have P. yoelii CS and GFP coding sequences as transgenes. In addition to these transgenes, a P. yoelii CS protein-derived B cell (PyCS-B) epitope - (QGPGAP $)_{3}$ - was inserted into hypervariable region 5 (HVR5) of the hexon (between 268 and 269 aa) or the HI loop of the fiber (between 543 and 544 aa) to make B-HVR5/CS-GFP or B-Fib/CS-GFP, respectively (Table 1). For HVR1 modification, the HVR1 sequence (from 138 to 164 aa) of WT/CS-GFP was replaced with the PyCS-B epitope to make B-HVR1/CS-GFP (Table 1). SDSPAGE and Western blot analysis with anti-sporozoite antibody confirmed the PyCS-B epitope insertion in the targeted capsid proteins (Figure 2A). As expected, the intensity of bands in Western blotting correlated with the copy number of the capsid proteins where the epitope was inserted (fiber, 36 copies per virion; hexon, 720 copies per virion). The lower band in lane 4 in Western blotting appeared to be a degraded hexon, as previously reported (15).

To assess whether the PyCS-B epitope - (QGPGAP $)_{3}$ - was presented on Ad particle, the purified capsid-modified rAd particles were coated onto ELISA plate and detected with an anti-recombinant P. yoelii CS protein antibody that recognizes (QGPGAP) repeat. The antibody recognized all capsid-modified Ads, indicating that the PyCS-B epitope incorporated in capsid proteins was well exposed to the outside of Ad virions (Figure 2B). Interestingly, anti-(QGPGAP) ${ }_{3}$ antibody showed similar reactivity to fiber-modified Ad compared with that of the hexon-modified Ad by an ELISA assay, although the copy number of fiber was 20-times less than that of hexon. This is probably due to the spike-like structure of fiber, which might make the $\mathrm{B}$ epitope more accessible to the antibody.

All capsid-modified rAds showed similar P. yoelii CS transduction efficiency (Figure 3A), ratio of virus particle unit to infectious unit (Table 2), and infectious virus productivity (Figure 3B) as WT/CSGFP. These data indicated that the PyCS-B epitope insertion into HVR1, HVR5 of the hexon, or the HI loop of fiber does not affect Ad fitness, at least in vitro. Also, the capsid-modified Ad genome seems to be as stable as that of WT/CS-GFP, because no deletion of the inserted epitope sequence from the Ad genome was detected, even after 15 passages of capsid-modified Ad (Figure 3C).

To evaluate the effect of incorporating the PyCS-B epitope in capsid proteins on infectivity, AD293 cells, mouse bone marrow DCs, CAR/A20 cells, and CAR/L1210 cells were infected with capsid-modified Ad in vitro. CAR/A20 and CAR/L1210 are mouse cell its B cell epitope on surface, we measured cellular and humoral responses against the $P$. yoelii $\mathrm{CS}$ protein at 2 weeks after a single immunization with the capsid-modified Ad, at doses ranging from $1 \times 10^{8}$ to $1 \times 10^{10}$ virus particles (v.p.) (Figure $5 \mathrm{~A}$ ). Both humoral and cellular responses to the P. yoelii CS transgene product, namely the anti-(PPQQ $)_{4}$ antibody response and $P$. yoelii $\mathrm{CS}-$-specific $\mathrm{CD}^{+} \mathrm{T}$ cell response, induced by capsid-modified Ad - except those induced by fiber-modified Ad (B-Fib/CS-GFP) - were somewhat lower than those induced by WT/CS-GFP Ad (Figure 5, B and C). However, the levels of anti-(QGPGAP) 3 antibody response induced by capsid-modified Ad were similar to those induced by WT/CS-GFP, most likely due to the surface expression of (QGPGAP) ${ }_{3}$ epitope by capsid-modified Ad. A single immunization with WT/GFP at any dosage failed to induce a significant level (<10 spots $/ 10^{6}$ splenocytes) of $P$. yoelii CS-specific $\mathrm{CD}^{+} \mathrm{T}$ cells secreting IFN- $\gamma$ (data not shown).

In the next set of experiments, mice were given multiple doses of capsid-modified Ad, with increasing doses (i.e., $1 \times 10^{8}, 1 \times 10^{9}$, and $1 \times 10^{10}$ v.p.) at 3-week intervals, as shown in Figure 6A. With this immunization regimen, both the levels of anti-(PPQQ) ${ }_{4}$ antibody titers (Figure 6B, bottom panels) and P. yoelii CS-specific $\mathrm{CD}^{+} \mathrm{T}$ cell response (Figure 6C) induced by capsid-modified Ad were significantly decreased compared with those induced by WT/ CS-GFP. However, all capsid-modified Ads were able to induce a significantly higher level of anti-(QGPGAP) ${ }_{3}$ antibody response

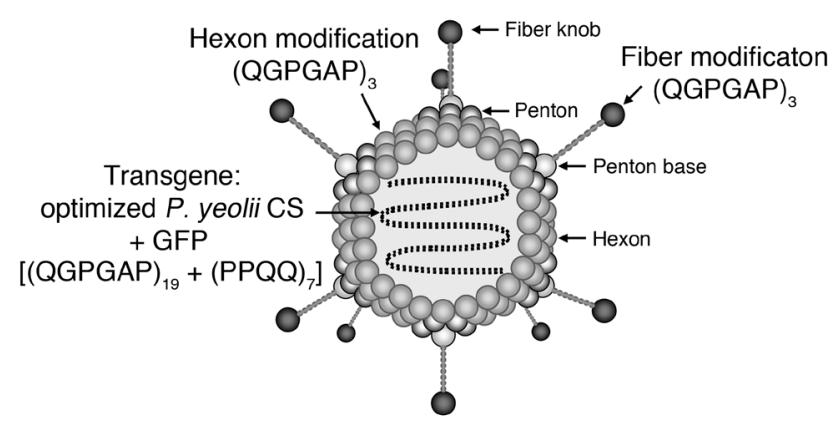

\section{Figure 1}

Structure of capsid-modified rAd. An immunodominant B cell epitope of $P$. yoelii CS protein (QGPGAP) ${ }_{3}$ was inserted in the hexon and/or in the fiber of WT/CS-GFP, which has optimized $P$. yoelii CS and GFP coding sequences as transgenes. $P$. yoelii CS protein contains 2 tandem repeats of (QGPGAP) ${ }_{19}$ and $(P P Q Q)_{7}$ in the central domain. 
A

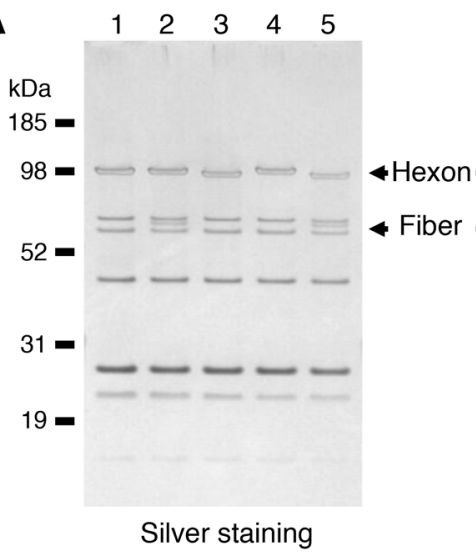

1
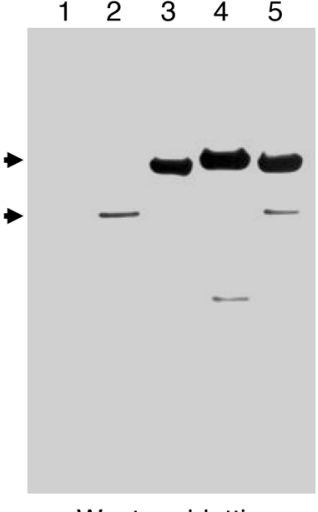

45

Western blotting
B

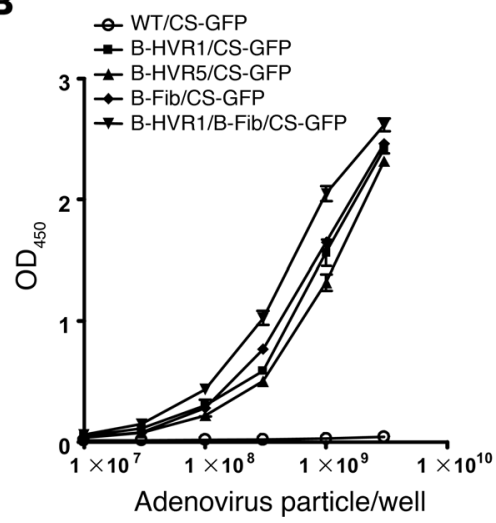

Figure 2

Validation of capsid-modified rAds. (A) Sliver staining and Western blotting of capsid-modified rAds. For Western blotting, the inserted epitope was detected with mouse polyclonal anti-sporozoite antibody. Lane 1, WT/CS-GFP; lane 2, B-Fib/CS-GFP; lane 3, B-HVR1/CS-GFP; lane 4, B-HVR5/CS-GFP; lane 5, B-HVR1/B-Fib/CS-GFP. (B) Presentation of the PyCS-B epitope on rAd virion surface. ELISA plates were coated with the indicated amounts of intact purified rAd virions and incubated with anti-P. yoelii CS antibody, followed by detection with HRP-labeled anti-mouse IgG antibody. Data are shown as mean \pm SD for triplicate wells.

than WT/CS-GFP at week 10 (Figure 6B, top panels). These results clearly indicated that the boosting effect of capsid-modified Ad on anti-(QGPGAP) ${ }_{3}$ antibody response was due to the surface expression of (QGPGAP) ${ }_{3}$ epitope by capsid-modified Ad.

More importantly, when we determined the vaccine efficacy of capsid-modified Ad by challenging the immunized mice with P. yoelii sporozoites and then determining the parasite burden in the liver by measuring $P$. yoelii rRNA, we found that vaccination with all capsid-modified Ads except B-HVR5/CS-GFP induced a higher level of protection than WT/CS-GFP, resulting in a significantly lower parasite burden in the malaria-challenged mice (Figure $6 \mathrm{D})$. To evaluate the effect of elevated anti-(QGPGAP) ${ }_{3}$ antibody titer induced by capsid-modified Ad on protection, a correlation analysis was done between the 2 parameters, anti-(QGPGAP) ${ }_{3}$ antibody titer and parasite burden in the liver. In the B-HVR1/CS-GFP, B-Fib/CS-GFP, or B-HVR1/B-Fib/CS-GFP immunized groups, mice that had higher anti-QGPGAP antibody titer appeared to be more protected (Figure 6E), suggesting that the enhancement of antimalarial protection in mice vaccinated with these capsid-modified Ads is likely due to the increased anti-(QGPGAP) ${ }_{3}$ antibody response.

Anti-sporozoite antibodies and their neutralizing ability. The nature of antibodies induced by capsid-modified Ad was evaluated in order to confirm that the augmented protection was mediated by malaria-specific antibody response. First, an indirect immunofluorescence assay (IFA) was performed to determine the antibody titers against whole parasites in the sera of capsid-modified Ad-immunized mice at week 10. We found that B-HVR1/CSGFP and B-HVR1/B-Fib/CS-GFP induced the highest IFA titer against sporozoites (Figure 7A), indicating that the insertion of PyCS-B epitope in the HVR1 of the hexon enabled Ad to elicit a robust antibody response against not only a synthetic peptide, but also a native epitope present in the parasites. Second, we performed an in vitro sporozoite neutralization assay using human CD81-expressing HepG2 cells as target cells, in order to determine whether mice vaccinated with capsid-modified Ad developed "protective" antibodies capable of neutralizing the infectivity of sporozoites. CD81 is a molecule that is necessary for malaria parasites to form parasitophorous vacuoles in hepatocytes in which they multiply and develop into schizonts (22), thus greatly increasing the in vitro infectivity of sporozoites. We found that the sera collected from mice immunized with capsid-modified Ads, particularly B-HVR1/CS-GFP and B-HVR1/B-Fib/CS-GFP, could almost completely inhibit (99\%) the sporozoite infectivity in vitro (Figure $7 \mathrm{~B})$. It is noteworthy that the degree of inhibition in this assay was inversely correlated with the IFA titers shown in Figure 7A and correlated with the level of protection shown in Figure 6D. All together these results indicate that multiple immunizing doses of capsid-modified Ads, particularly those expressing PyCS-B epitope in the HVR1 of the hexon, could elicit augmented antibody response that is reactive against native malarial parasites and can neutralize the infectivity of the parasites.

Sterile immunity elicited by capsid-modified $r A d$. Next, we tested whether immunization with capsid-modified rAd protects mice from developing a blood-stage malaria infection after sporozoite challenge. We performed the experiments twice, and in each experiment, $20 \mathrm{BALB} / \mathrm{c}$ mice in each group were immunized 3 times with WT/CS-GFP or B-HVR1/CS-GFP. At 4 weeks after the last immunization, the mice were intravenously challenged with 50 P. yoelii sporozoites. Giemsa-stained blood smears were analyzed from 3 to 12 days after challenge to detect blood-stage malaria

Table 2

Ratios of virus particle units to infectious units for capsidmodified rAds

\begin{tabular}{|c|c|c|}
\hline & \multicolumn{2}{|c|}{ Virus particle unit/infectious unit } \\
\hline & Experiment 1 & Experiment 2 \\
\hline WT/CS-GFP & 34.1 & 34.1 \\
\hline B-HVR1/CS-GFP & 46.5 & 31.6 \\
\hline B-HVR5/CS-GFP & 47.9 & 32.6 \\
\hline B-Fib/CS-GFP & 38.2 & 31.6 \\
\hline B-HVR1/B-Fib/CS-GFP & 46.5 & 38.4 \\
\hline
\end{tabular}


A

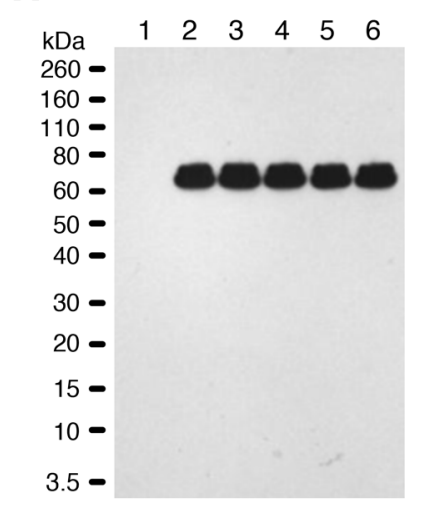

B

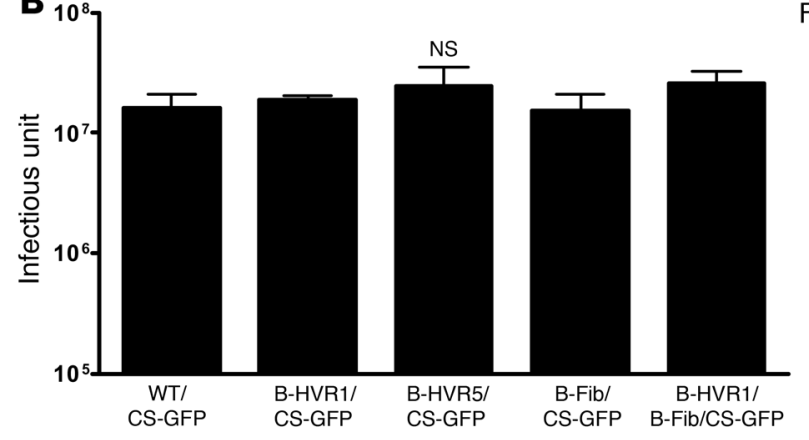

C
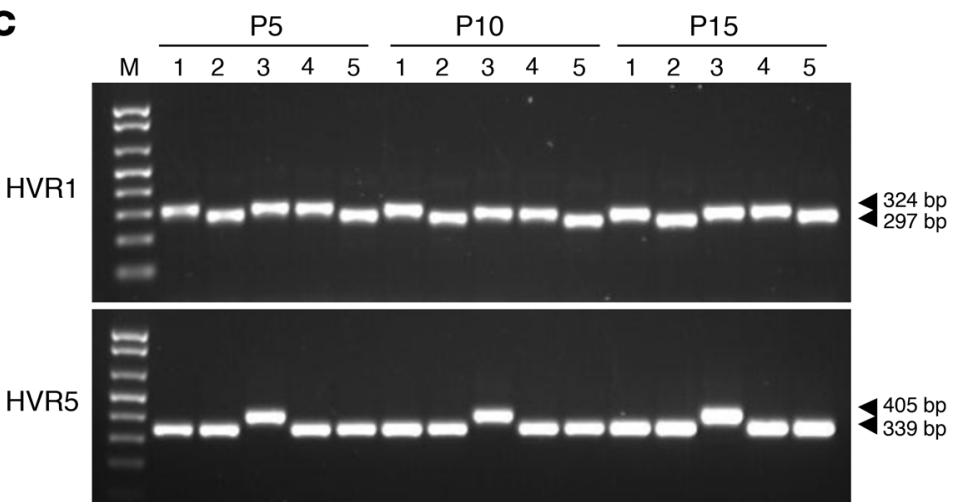

Fiber

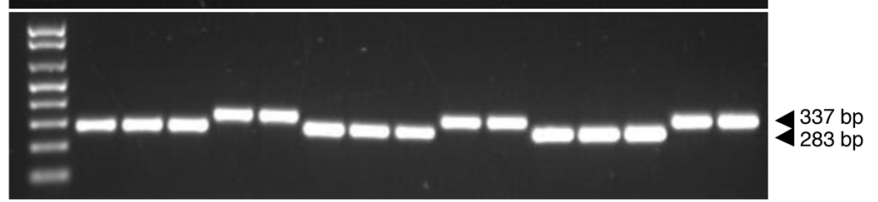

Figure 3

Transduction ability, productivity, and genetic stability of capsid-modified rAds. (A) P. yoelii CS protein expression after capsid-modified rAd infection of HeLa cells. Whole cell lysates were prepared 24 hours after infection, and $P$. yoelii CS protein expression was analyzed by Western blotting. P. yoelii CS protein was detected with mouse monoclonal anti-P. yoelii CS antibody (9D3). Lane 1, HeLa (uninfected); lane 2, WT/CSGFP; lane 3, B-Fib/CS-GFP; lane 4, B-HVR1/CS-GFP; lane 5, B-HVR5/CS-GFP; lane 6, B-HVR1/B-Fib/CS-GFP. (B) Productivity of capsidmodified rAds. Infectious units of the crude Ad solutions prepared from single-round infection of AD293 cells were determined using end point dilution method. Data are shown as mean \pm SD for triplicate wells. (C) Stability of the inserted PyCS-B epitope sequence in Ad genome after Ad passages. $5 \times 10^{5}$ AD293 cells in 24-well plates were infected with $1 \times 10^{8} \mathrm{v}$.p. of capsid-modified rAd, and crude Ad solution was prepared by freeze-thaw cycles 2 days after infection. One-tenth of the crude virus solution was used for the next round of infection. Ad genome corresponding to HVR1, HVR5 of the hexon, or HI loop of fiber was amplified by PCR, using crude Ad solutions as templates after indicated passage of capsid-modified rAds. P5, P10, and P15 represent crude Ad solutions after 5, 10, and 15 passages, respectively. M, 1-kb plus marker (Invitrogen). Lane 1, WT/CS-GFP; lane 2, B-HVR1/CS-GFP; lane 3, B-HVR5/CS-GFP; lane 4, B-Fib/CS-GFP; lane 5, B-HVR1/B-Fib/CS-GFP.

parasite infection. When experiments 1 and 2 were combined, 30 out of 40 mice (75\%) were infected in the WT/CS-GFP-immunized group, whereas 35 out of 40 mice $(87.5 \%)$ were infected in the naive group (Table 3). B-HVR1/CS-GFP-immunized mice were significantly ( $P=0.014$ by Fisher's exact test) more protected than WT/ CS-GFP-immunized mice; only 15 out of 40 of these mice (37.5\%) were infected, which is consistent with the result of the protection experiment, measured by parasite burden in liver (Figure 6D).

P. falciparum CS-specific humoral response induced by capsid-modified Ad expressing P. falciparum CS. To determine whether the results we obtained using a rodent malaria parasite would hold for a human malaria model, we constructed a capsid-modified rAd using the P. falciparum CS protein. P. falciparum is the most virulent parasite among the human malaria species and the main target of malaria vaccine development. The immunodominant central repeat sequence in $P$. falciparum CS protein is NANP-repeat; therefore, we inserted (NANP) $)_{4}$ into the HVR1 of the hexon to construct NANP-HVR1/P. falciparum CS (Table 1). Immunization of mice with 3 doses of NANP-HVR1/P. falciparum CS enhanced antiNANP antibody titer more than 100 fold (Figure 8). This indi- cated that the effect of capsid-modified Ad on humoral immune response is not epitope sequence specific and that this modified capsid vaccine platform can most certainly be applied to human malaria vaccine development.

Effect of capsid-modification on anti-Ad immunity. In order to test whether hexon modification could affect neutralization of Ad by anti-Ad5 antibody, AD293 cells were infected with each capsid-modified rAd in the presence of serum from naive mice or mice immunized with replication-incompetent WT Ad5 (WT/Empty) twice. Ad infection was monitored by measuring GFP expression using flow cytometric analysis. Replacement of HVR1 with the PyCS-B epitope clearly made the Ad resilient to anti-Ad5 serum, whereas the modification of HVR5 or fiber had no effect (Figure 9A). Interestingly, mouse anti-P. yoelii CS protein antibodies neutralized only hexonmodified rAds, namely B-HVR1/CS-GFP, B-HVR5/CS-GFP, and B-HVR1/B-Fib/CS-GFP (Figure 9A), indicating that blocking of hexon by antibodies efficiently neutralizes Ad infection.

Next, we used human sera in this assay to see whether similar observations could be made in humans naturally exposed to Ad5 infection. We first screened 18 sera samples from people of Euro- 

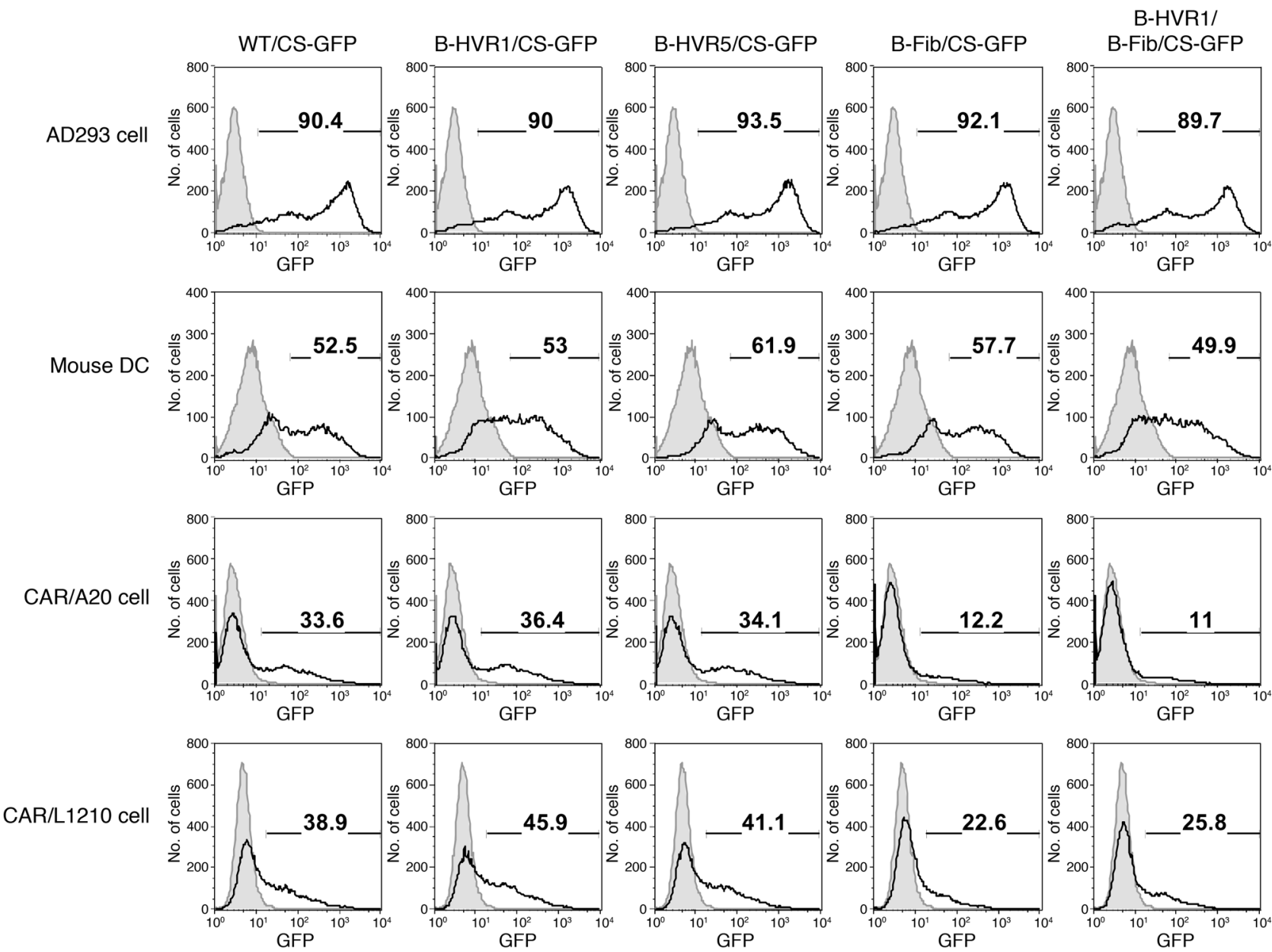

Figure 4

Infectivity of capsid-modified rAds in vitro. AD293 cells, DCs, CAR/A20 cells, or CAR/L1210 cells were seeded in a 48-well plate $\left(2 \times 10^{5}\right.$ cells/ well), and then capsid-modified or unmodified rAd was added at a ratio of 1:250, 1:50,000, 1:1,000, or 1:600 (cell/rAd particle), respectively. After 24-hour incubation, cells were analyzed by flow cytometry to measure GFP expression. Filled areas represent cells without rAd, and solid lines represent cells with $\mathrm{rAd}$. The numbers indicate the percentage of cells that stain positive by a flow cytometric analysis.

pean descent and found that 5 out of 18 sera samples showed a strong neutralizing activity against WT Ad5 (data not shown). We then assessed the susceptibility of capsid-modified Ad to these 5 selected human sera samples in the same assay described above. We found that B-HVR1/CS-GFP and B-HVR1/B-Fib/CS-GFP exhibited the highest infectivity to target cells, in spite of the presence of the human anti-Ad5 neutralizing sera (Figure 9B), confirming HVR1 as a major target of neutralizing anti-Ad5 antibodies in humans.

Effect of in vivo anti-Ad immunity on the immunogenicity of capsid-modified Ad. Last, we sought to determine whether HVR1 is a critical target for preexisting anti-Ad immunity in vivo by modifying the HVR1 of rAd. For this purpose, mice were infected with WT/Empty Ad twice to mount sufficient preexisting anti-Ad immunity (Figure $10 \mathrm{~A}$ ) and randomized based on their anti-Ad antibody titers, as determined by ELISA (Figure 10B). The mice were then given a single immunizing dose of capsid-modified rAd, and the level of $P$. yoelii $\mathrm{CS}$ antigen-specific $\mathrm{CD} 8^{+} \mathrm{T}$ cell response was measured 2 weeks after the priming. As expected, vaccination with all rAds induced a similar level of $P$. yoelii $\mathrm{CS}$ antigen-specific $\mathrm{CD}^{+} \mathrm{T}$ cell response in naive mice (Figure 10C); whereas in Ad-immune mice, only vaccination with B-HVR1/CS-GFP or B-HVR1/B-Fib/CS-GFP was able to induce a level of $P$. yoelii $\mathrm{CS}$ antigen-specific $\mathrm{CD}^{+} \mathrm{T}$ cell response significantly greater than that of other capsid-modified Ads or unmodified Ad (Figure 10D). More importantly, we have also measured the level of antibody response against (QGPGAP) 3 epitope, which is expressed on the capsid proteins of rAd, in mice infected with WT/Empty Ad, followed by vaccination with capsidmodified rAd (Figure 11A). We found that only mice vaccinated with B-HVR1/CS-GFP and B-HVR1/B-Fib/CS-GFP were able to mount a significantly higher titer of anti-(QGPGAP) ${ }_{3}$ antibody than those vaccinated with other rAds (Figure 11B). Taken together, these results indicate that only the modification of HVR1, but not other portions of the capsid proteins, could circumvent preexisting anti-Ad5 immunity, while maintaining the immunogenicity of a B cell epitope of the P. yoelii CS protein expressed on the capsid and also that of a P. yoelii CS antigen expressed as a transgene.

Finally, we determined the neutralizing activity against Ads of sera from mice immunized with 2 doses of WT/GFP, WT/CS-GFP, or B-HVR1/B-Fib/CS-GFP. As shown in Figure 11C, pooled serum from mice immunized with WT/GFP or WT/CS-GFP could neu- 
A
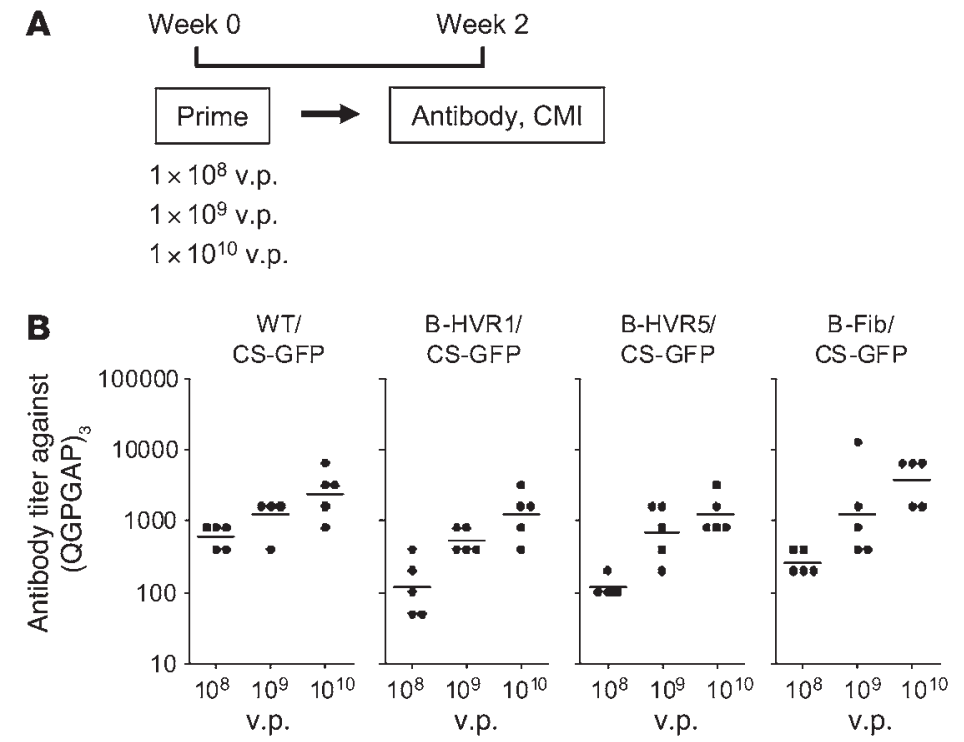
B-HVR1/B-Fib/ CS-GFP
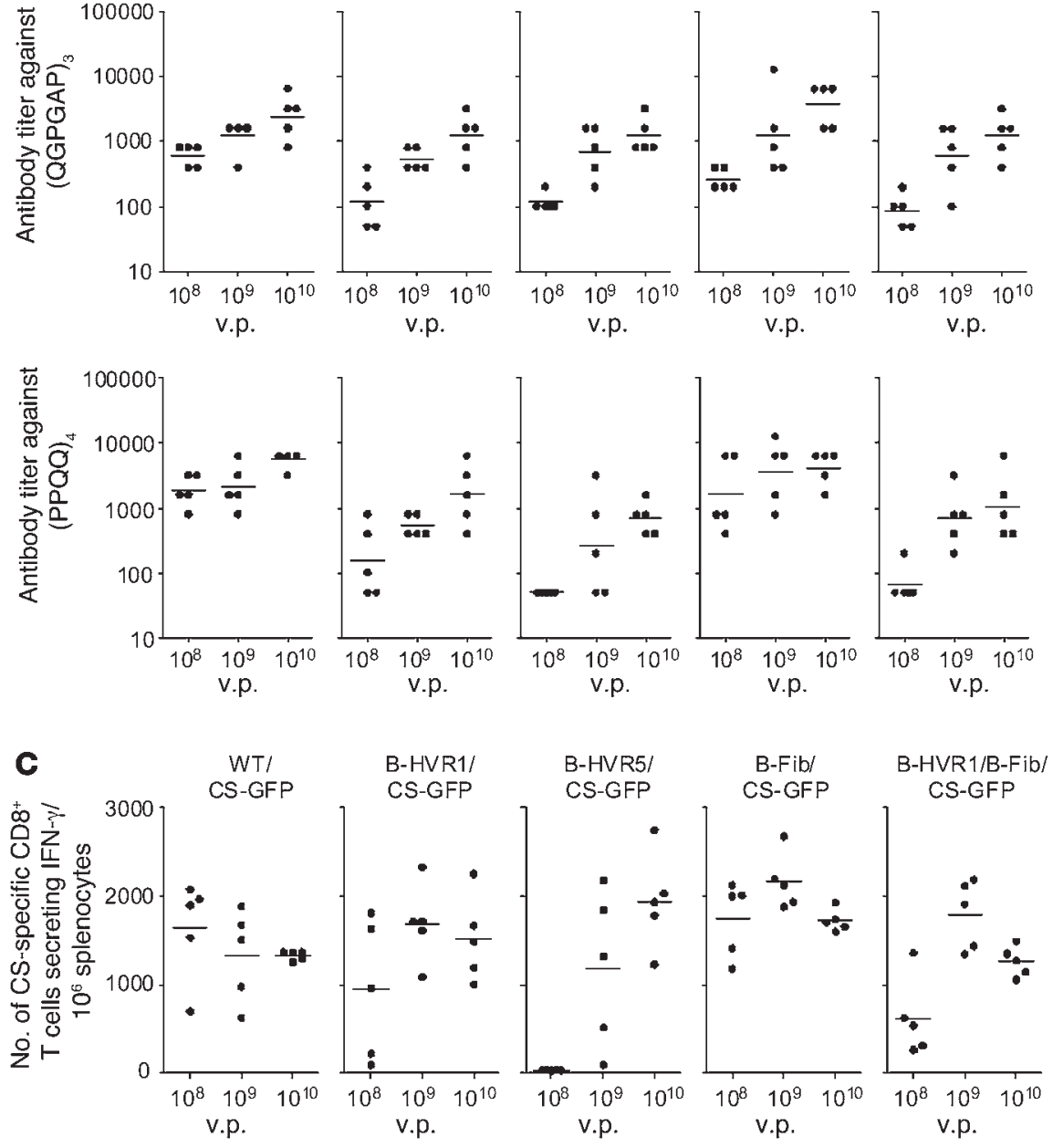

Figure 5

$P$. yoelii CS-specific immune responses after a single immunizing dose of capsid-modified rAd. (A) Immunization regimen. Groups of naive BALB/c mice (5 per group) were immunized with $1 \times 10^{8}, 1 \times 10^{9}$, or $1 \times 10^{10}$ v.p. of various rAds intramuscularly, and $P$. yoelii CS-specific humoral and cell-mediated immune responses (CMI) were measured at 2 weeks after immunization. (B) Anti$(\mathrm{QGPGAP})_{3}$ and anti-(PPQQ) $)_{4}$ responses. Antibody titer was determined using an end point dilution titer by ELISA. Horizontal bars represent the geometric mean. (C) $P$. yoelii CS-specific CD8 ${ }^{+} \mathrm{T}$ cell response. $P$. yoelii CS-specific $\mathrm{CD}^{+} \mathrm{T}$ cell responses were determined using IFN- $\gamma$ ELISPOT assay. Horizontal bars represent the mean. tralize Ad with intact HVR1. However, serum from mice immunized with B-HVR1/B-Fib/CS-GFP was unable to neutralize WT/ CS-GFP but strongly neutralized HVR1-modified Ad (Figure 11C). This pooled serum partially neutralized other capsid-modified rAds. These results collectively indicate that HVR1 region of the hexon is the most critical target epitope for both the induction of and the susceptibility to neutralizing anti-Ad5 antibody.

\section{Discussion}

Ad has been shown to be a promising vaccine vector for various infectious diseases, including malaria (23), because of its unique characteristic ability to induce a potent antigen-specific CD8 ${ }^{+}$ $\mathrm{T}$ cell response. However, Ad-based vaccines have been shown to induce a relatively weak humoral response against a transgene, and preexisting immunity to Ad, which is highly prevalent among the general population, hampers the immunogenicity of any Ad-based vaccine. In order to overcome these weaknesses, we constructed what we believe to be a novel Ad-based malaria vaccine by inserting an immunodominant B cell epitope of the P. yoelii CS protein, PyCS-B epitope, into Ad capsid proteins to present the antigen to the outside of the Ad virion. Multiple doses of vaccination with the capsid-modified Ad not only augmented a humoral response against PyCS-B epitope expressed in the capsid but also induced a significantly increased level of protection against subsequent malaria challenge. In addition, replacement of HVR1 with the PyCS-B epitope circumvented neutralization by preexisting antiAd antibody and maintained the immunogenicity of the B cell epitope expressed in the capsid.

In order to dissect immune responses induced by capsid-modified Ad, we measured P. yoelii $\mathrm{CS}-$-specific $\mathrm{CD}^{+} \mathrm{T}$ cell response and humoral response to (QGPGAP) $)_{3}$ or (PPQQ) ${ }_{4}$ epitope, respectively. This is an excellent strategy to evaluate the effect of PyCS-B epit- 
A

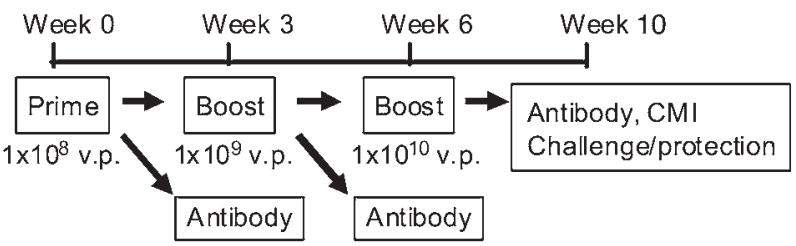

B
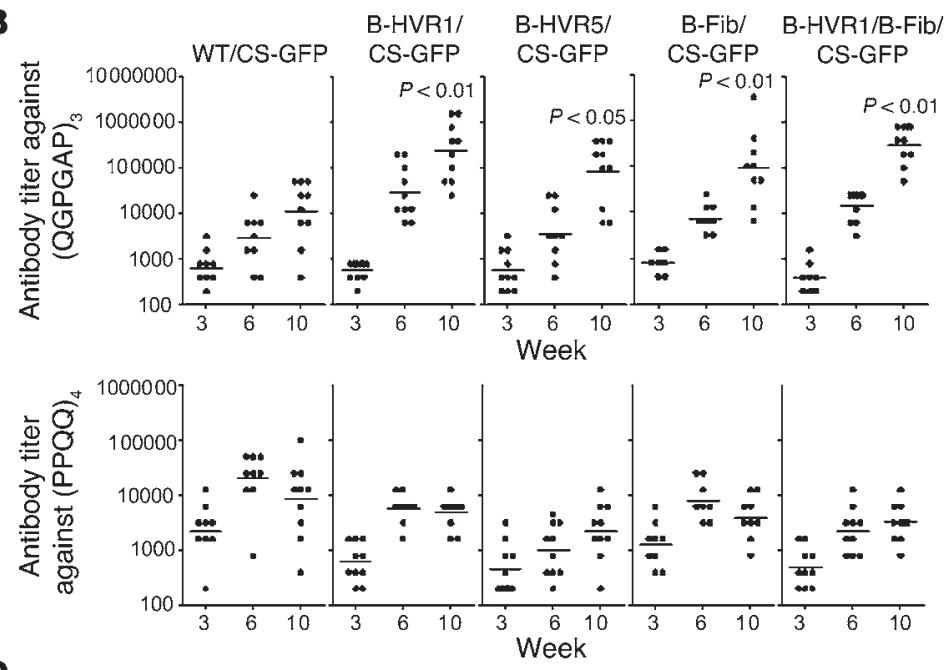

C

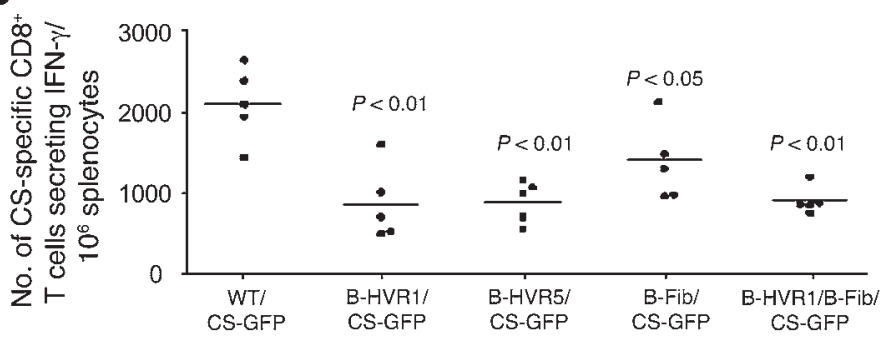

D

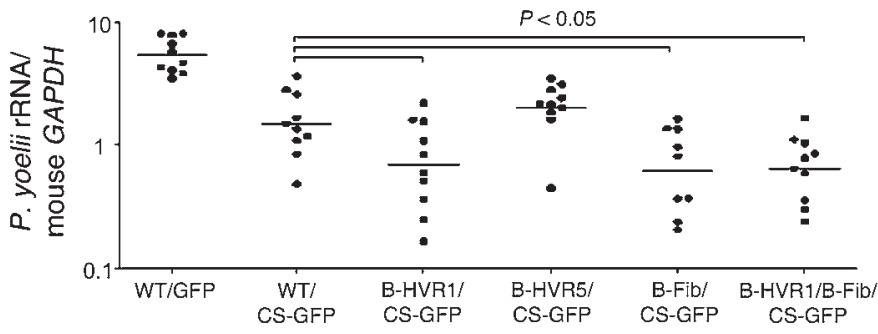

E

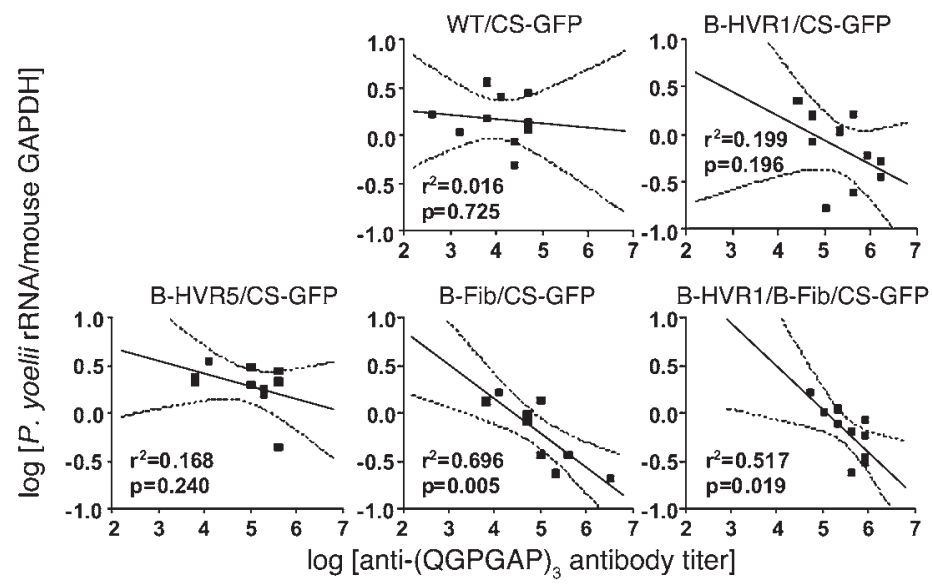

Figure 6

$P$. yoelii CS-specific immune responses and antimalarial protection after multiple immunizing doses of capsid-modified rAd. (A) Immunization regimen. Groups of naive BALB/c mice were immunized intramuscularly with $1 \times 10^{8} \mathrm{v}$.p. of various rAds at week $0,1 \times 10^{9}$ of various rAds at week 3 , and $1 \times 10^{10}$ of various rAds at week 6 , and $P$. yoelii CSspecific humoral and cell-mediated immune response were measured at the time indicated. (B) Anti-(QGPGAP) ${ }_{3}$ and anti-(PPQQ $)_{4}$ humoral responses. Antibody titers were determined by ELISA. Antibody titers below 100 were plotted as 50 . Horizontal bars represent the geometric mean. (C) $P$. yoelii CS-specific $\mathrm{CD}^{+} \mathrm{T}$ cell response. $P$. yoelii CS-specific CD8 ${ }^{+} \mathrm{T}$ cell response in spleen was evaluated with IFN- $\gamma$ ELISPOT assay at week 10. Data are shown as the number of IFN- $\gamma$-secreting $P$. yoelii CS-specific T cells in 1 million splenocytes. Horizontal bars represent the mean. (D) Antimalarial protection. The same groups of immunized mice in B were challenge with $2 \times 10^{4}$ infectious $P$. yoelii sporozoites via tail vein. Forty-two hours after the challenge, parasite burden in liver was determined by quantifying parasite ribosomal RNA with real-time PCR. For statistical analysis, the values were log-transformed, and then 1-way ANOVA followed by a Dunnett's test was used. Horizontal bars represent the geometric mean. (E) Correlation between anti-QGPGAP antibody titer and parasite burden in liver. Log-transformed anti-(QGPGAP) $)_{3}$ antibody titer at week 10 and log-transformed parasite burden in liver in each mouse is scatter-plotted to evaluate the correlation between these variables. The solid lines show linear regression curves, and the dotted lines show $95 \% \mathrm{Cl}$ of the curves.

ope insertion on immune response, because the CD8 ${ }^{+}$ $T$ cell epitope, SYVPSAEQI, and PPQQ repeat are present only in the transgene product, whereas (QGPGAP) ${ }_{3}$ epitope is present both in the capsid protein and transgene product. After a single immunizing dose of each capsidmodified Ad and unmodified Ad, all capsid-modified Ads induced a lower SYVPSAEQI-specific $\mathrm{CD}^{+} \mathrm{T}$ cell response and anti-(PPQQ) ${ }_{4}$ antibody response, indicating that in vivo infectivity of rAd was somewhat reduced by capsid-modification. Among those, fiber modification had the least impairment in inducing these responses, most likely because fiber-modified rAd could retain in vivo infectivity comparable with that of WT/ CS-GFP. Surprisingly, these observations are not consistent with Ad infectivity in vitro. In our experiment, there was no difference between hexon-modified Ad and WT/CS-GFP in infectivity to AD293 cells, mouse DCs, and CAR-expressing A20 and L1210 cells in vitro. Fiber modification, in contrast, slightly affected CAR-dependent Ad infection in vitro. Interaction between CAR and fiber (21) may not play an important role in inducing a transgene-specific immune response in vivo, at least in a mouse model. It is well-known that Ad infectivity in vitro does not always correlate with the in vivo infectivity $(24,25)$. In this regard, establishing an in vitro assay that correlates with the ability of Ad to induce immune response in vivo is required for future development of capsid-modified Ad-based vaccine.

After multiple immunizing doses of $\mathrm{rAd}$, all capsidmodified Ads still induced a lower $\mathrm{CD}^{+} \mathrm{T}$ cell response 

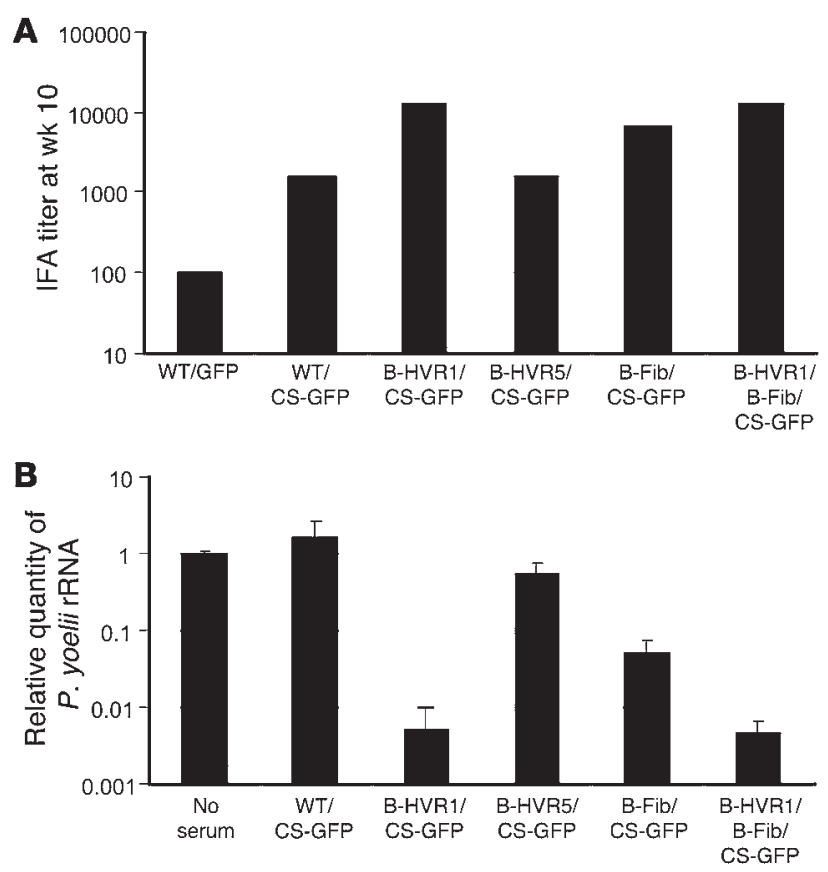

and humoral response to the transgene product. This is partly due to the lower infectivity of capsid-modified rAd as described above and in part due to the neutralizing antibody raised upon multiple immunization against respective rAds, i.e., anti-P. yoelii CS antibody that neutralizes HVR1- and HVR5-modified rAds and/or antiAd5 antibody that neutralizes HVR5- and fiber-modified rAds, as shown in Figure 9A. Most importantly, we found that among the sites tested in this study, HVR1 is the best for PyCS-B cell epitope insertion to induce not only (QGPGAP) ${ }_{3}$ epitope-specific antibody but also protective antibody against the malaria parasite, which would ultimately mediate the protection against malaria. When protection was evaluated in the P.yoelii sporozoite challenge model, immunization with B-HVR1/CS-GFP, B-Fib/CS-GFP, and B-HVR1/ B-Fib/CS-GFP all significantly reduced parasite burden in liver after challenge compared with WT/CS-GFP, and in particular, immunization with B-HVR1/CS-GFP also protected mice from blood-stage malaria infection more than WT/CS-GFP. We found that the augmented protection by B-HVR1/CS-GFP, B-Fib/CSGFP, or B-HVR1/B-Fib/CS-GFP appears to correlate with robust anti-(QGPGAP) ${ }_{3}$ antibody response. Also, serum from mice immunized with B-HVR1/CS-GFP and HVR1/B-Fib/CS-GFP recognized and neutralized sporozoites strongly in vitro. These observations have led us to conclude that (QGPGAP) ${ }_{3}$ epitope-specific humoral response plays a key role in preventing sporozoite infection by capsid-modified Ad. The requirement of high anti-(QGPGAP) ${ }_{3}$ antibody titer for protection against malaria in our study is consistent with the results of other studies $(26,27)$. The superior protective efficacy induced by HVR1-modified rAd was confirmed by the experiments in which blood infection was measured by the presence of parasitemia in thin blood smears upon sporozoite challenge, as shown in Table 3 . We must note that although vaccination with B-HVR1/CS-GFP significantly increased the number of mice protected from getting blood-stage malaria infection compared with that of WT/CS-GFP, this HVR1 modification could not induce sterile immunity in all mice vaccinated. In order to further

\section{Figure 7}

Recognition and neutralization of $P$. yoelii sporozoites by serum from immunized mice. (A) Groups of naive BALB/c mice (5 per group) were immunized intramuscularly with $1 \times 10^{8} \mathrm{v}$.p. of various rAds at week 0 , $1 \times 10^{9} \mathrm{v}$.p. of various rAds at week 3 , and $1 \times 10^{10} \mathrm{v}$.p. of various rAds at week 6 , and then serum samples were prepared at week 10. IFA was done to determine anti-sporozoite antibody titers, using pooled serum samples at week 10. IFA titers were determined as the highest dilution producing fluorescence under a fluorescent microscope. Serum from WT/GFP was negative at the lowest dilution (200-fold dilution), and its titer is described as 100. (B) Neutralization of $P$. yoelii sporozoites in vitro. Sporozoites were incubated with CD81/HepG2 for 2 hours in the presence of 30 -fold diluted pooled serum described in A. After incubation, uninfected sporozoites were washed out, and then the cells were cultured for 42 hours. The relative amount of parasite ribosomal RNA to human GAPDH mRNA was measured by real-time $P C R$. Data are shown as the mean \pm the SD for triplicate cultures.

improve the vaccine efficacy, we are currently seeking an adjuvant that can enhance the immunogenicity of HVR1-modified Ad. In parallel, in order to increase the Th cell function, we are constructing a HVR1-modified Ad that will express the $\mathrm{CD}^{+} \mathrm{T}$ cell epitope of the P. yoelii CS antigen in the capsid proteins and/or nucleoproteins of WT/CS-GFP.

NANP-HVR1/P. falciparum CS, which has (NANP) ${ }_{4}$ from the immunodominant central repeat region of $P$. falciparum $C S$ protein, also induced more than 100-times higher anti-NANP antibody titer than WT/P. falciparum CS in the same immunization regimen. Together with the observations in the P. yoelii challenge model, we have shown that capsid-modified rAd would be one of the most promising human malaria vaccine platforms studied so far.

With regards to evading existing Ad immunity, an epitope mapping study of the neutralizing anti-Ad68 monoclonal antibodies suggested that HVR1 could be a critical epitope for the Adneutralizing antibody (28). Our HVR1-modified rAd, in which HVR1 was replaced with the PyCS-B epitope, became resistant

\section{Table 3}

Immunization of mice with capsid-modified rAds protects against development of blood-stage malaria infection

\begin{tabular}{|c|c|c|}
\hline Immunization & $\begin{array}{l}\text { No. of mice } \\
\text { challenged }\end{array}$ & $\begin{array}{l}\text { No. of mice } \\
\text { infected (\%) }\end{array}$ \\
\hline \multicolumn{3}{|l|}{ Experiment 1} \\
\hline None & 20 & $18(90 \%)$ \\
\hline WT/CS-GFP & 20 & $14(70 \%)$ \\
\hline B-HVR1/CS-GFP & 20 & $10(50 \%)$ \\
\hline \multicolumn{3}{|l|}{ Experiment 2} \\
\hline None & 20 & $17(85 \%)$ \\
\hline WT/CS-GFP & 20 & $16(80 \%)$ \\
\hline B-HVR1/CS-GFP & 20 & $5(25 \%)$ \\
\hline \multicolumn{3}{|l|}{ Combined } \\
\hline None & 40 & $35(87.5 \%)$ \\
\hline WT/CS-GFP & 40 & $30(75 \%)^{A}$ \\
\hline B-HVR1/CS-GFP & 40 & $15(37.5 \%)^{A}$ \\
\hline
\end{tabular}

AFor the statistical analysis of protection from blood-stage malaria infection, Fisher's exact test was applied to the combined data of experiments 1 and 2 (WT/CS-GFP vs. B-HVR1/CS-GFP). Immunization with B-HVR1/CS-GFP significantly reduced the number of mice infected with blood-stage malaria compared with WT/CS-GFP $(P=0.014)$. 


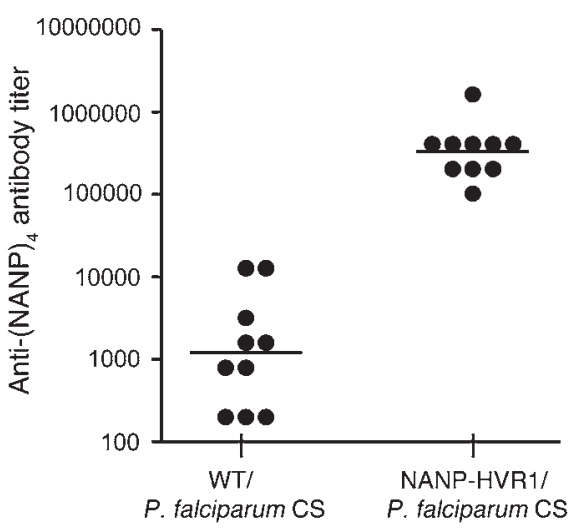

Figure 8

Augmented humoral immune response by (NANP) $)_{4}$-epitope insertion into HVR1 of the hexon. Groups of naive BALB/c mice (10 per group) were immunized intramuscularly with $1 \times 10^{8} \mathrm{v}$.p. of rAd at week 0 , $1 \times 10^{9}$ v.p. of rAd at week 3 , and $1 \times 10^{10}$ v.p. of rAd at week 6 , and then serum samples were prepared at week 9 . Anti-NANP antibody titer was determined using an ELISA end point dilution assay, using $(\mathrm{T} 1 \mathrm{~B})_{4}$ peptide for coating. Horizontal bars represent the geometric mean.

to neutralization by mouse and human anti-Ad sera, while being neutralized by anti-PyCS-B epitope antibody in vitro. Moreover, HVR1-modified rAd induced a higher level of both anti-(QGPGAP $)_{3}$ antibody response and $P$. yoelii $\mathrm{CS}$ antigen-specific $\mathrm{CD}^{+}$ $\mathrm{T}$ cell response in mice that mount preexisting anti-Ad immunity. These results clearly showed that HVR1 is one of the more crucial targets of preexisting immunity to Ad5. The reason for this might be that HVR1 is one of the most immunodominant $\mathrm{B}$ epitopes in the hexon. It is noteworthy that these results appear to be contradictory to those of the study by Roberts et al., which reported the replacement of HVR1 with that of Ad48 cannot circumvent preexisting anti-Ad5 immunity (20). However, if we look closely at the experimental procedure by Roberts et al., mice with a high level of preexisting anti-Ad5 immunity were vaccinated with a low dose $\left(10^{7}-10^{9}\right.$ v.p.) of HVR-modified Ad, leading to the conclusion that the replacement of HVR1 failed to evade the preexisting immunity. When we vaccinated mice that have preexisting anti-Ad5 immunity with a high dose $\left(10^{10} \mathrm{v} . p.\right)$ of HVR1-modified Ad expressing a malaria antigen, we observed a substantially lower level of a malaria antigen-specific $\mathrm{CD}^{+} \mathrm{T}$ cell response (Figure 10, C and D), indicating that HVR1 modification still could not fully elude anti-Ad5 immunity. However, we have also shown that among all our modifications with respect to HVR1, HVR5, and fiber protein, HVR1 modification circumvented anti-Ad5 immunity the most, thereby indicating HVR1 as one of the particularly critical targets of preexisting immunity to Ad5. This conclusion was corroborated by the fact that when anti-P. yoelii CS antibody was used to neutralize rAd in vitro, it neutralized equally both HVR1- and HVR5-modified rAds, suggesting that an epitope present in HVR5 can also be a target of neutralizing antibody, albeit to a lesser degree. The observation of HVR5 being the possible target of neutralizing antibody in addition to HVR1 is not contradictory to the study by Roberts et al., which reported that replacement of all HVRs with those of Ad48 was necessary to completely circumvent preexisting immunity to Ad5 (20), or to the study by Abe et al., which showed that
HVR5 modification could also circumvent preexisting anti-Ad5 immunity, although they never compared the effect of HVR5 modification with that of HVR1 modification (29).

In our study, the percentage of neutralizing antibody against HVR1 within all neutralizing antibodies seems to differ among human sera, and this variation could arise from the difference in immunogenicity of HVRs among individuals. Interestingly, no cross neutralization activity to HVR1-modified Ad was observed in mouse serum immunized with unmodified Ad in vitro and vice versa. This observation suggested that HVR1-modified Ad can be used in a heterologous prime-boost vaccination regimen with capsid-unmodified Ad.

Overall, our current study demonstrates attractive features of what we believe to be a novel capsid-modified Ad as a next-generation vaccine platform against malaria. This paper shows for the first time to our knowledge that a replacement of HVR1 with a $B$ cell epitope of a pathogen results in enhanced protective immunity against the pathogen by augmenting anti-B cell epitope antibody response and at the same time, circumvents preexisting antiAd immunity. The improved immunogenicity and vaccine efficacy of HVR1-modifed rAd, in spite of the presence of preexisting antiAd5 immunity, is likely to break new ground with regard to the rationale of the future designs of virus-based vaccines against not only malaria, but also other major infectious diseases of the world.

\section{Methods}

Cell lines. A sub-cell line of HEK293, AD293, was obtained from Stratagene. A20 cells (mouse B cell lymphoma line), L1210 cells (mouse lymphocytic leukemia cell line), HeLa cells (human cervical adenocarcinoma cell line), and HepG2 cells (human hepatocarcinoma cell line) were from ATCC. AD293, HeLa, and HepG2 cells were cultured in DMEM supplemented with $10 \%$ FBS and antibiotics. A20 and L1210 cells were in RPMI1640 supplemented with $10 \% \mathrm{FBS}$, antibiotics, and $55 \mu \mathrm{M} 2$-mercaptoethanol.

$r A d s$. The rAd vector used in this study was replication-defective, E1- and E3-deleted Ad5 (Stratagene). Capsid-modified Ad was constructed to have 2 CMV-derived expression cassettes for P. yoelii CS and GFP in the E1-deleted region, in addition to PyCS-B epitope (QGPGAP) $)_{3}$ insertion into the hexon of HVR1, HVR5, and/or fiber HI loop. For the purpose of evaluating infectivity of capsid-modified rAd, Ad shuttle vector pShuttle-CMV (Stratagene) was modified by inserting GFP expression cassette under the cloning sites. First, BsmBI-SacI fragment (pCMV-GFP) and SacI-BsmBI fragment (SV40 poly A signal) of pmaxGFP (Lonza) were blunted and inserted into the blunted SalI and KpnI sites of PUC19, respectively. The BamHI-EcoRI fragment of the resulting PCMV-GFP/PUC19 was inserted into the same sites of SV40pA/pUC19 to create the SV40pA-pCMV-GFP fragment. The fragment was blunted and inserted into the EcoRV site of pShuttle-CMV. The resulting shuttle vector (pShuttle-CMV-GFP) has dual pCMV promoters and SV40 pA signals for P. yoelii CS and GFP.

The P. yoelii CS gene was codon-optimized, except for the QGPGAP repeats, by overlapping PCR reaction, based on the JCat codon optimization algorithm (30). The optimized P. yoelii CS fragment, without the GPI-anchored motif, was inserted into KpnI and HindIII sites of pShuttleCMV-GFP. The resulting shuttle vector was used for homologous recombination with AdEasy-1 (Stratagene) in E. coli strain BJ5183 (Stratagene) to generate the rAd genome.

For fiber modification, the SpeI-PacI fragment of AdEasy-1 was subcloned into EcoRI and PstI sites of pUC19, using EcoRI-PacI and PstISpeI linker oligomers. To insert a PyCS-B cell epitope sequence into the $\mathrm{HI}$ loop of the fiber knob, the region containing EcoNI and MfeI sites was amplified by 2 -step PCR, using primers that have the epitope sequence 
A
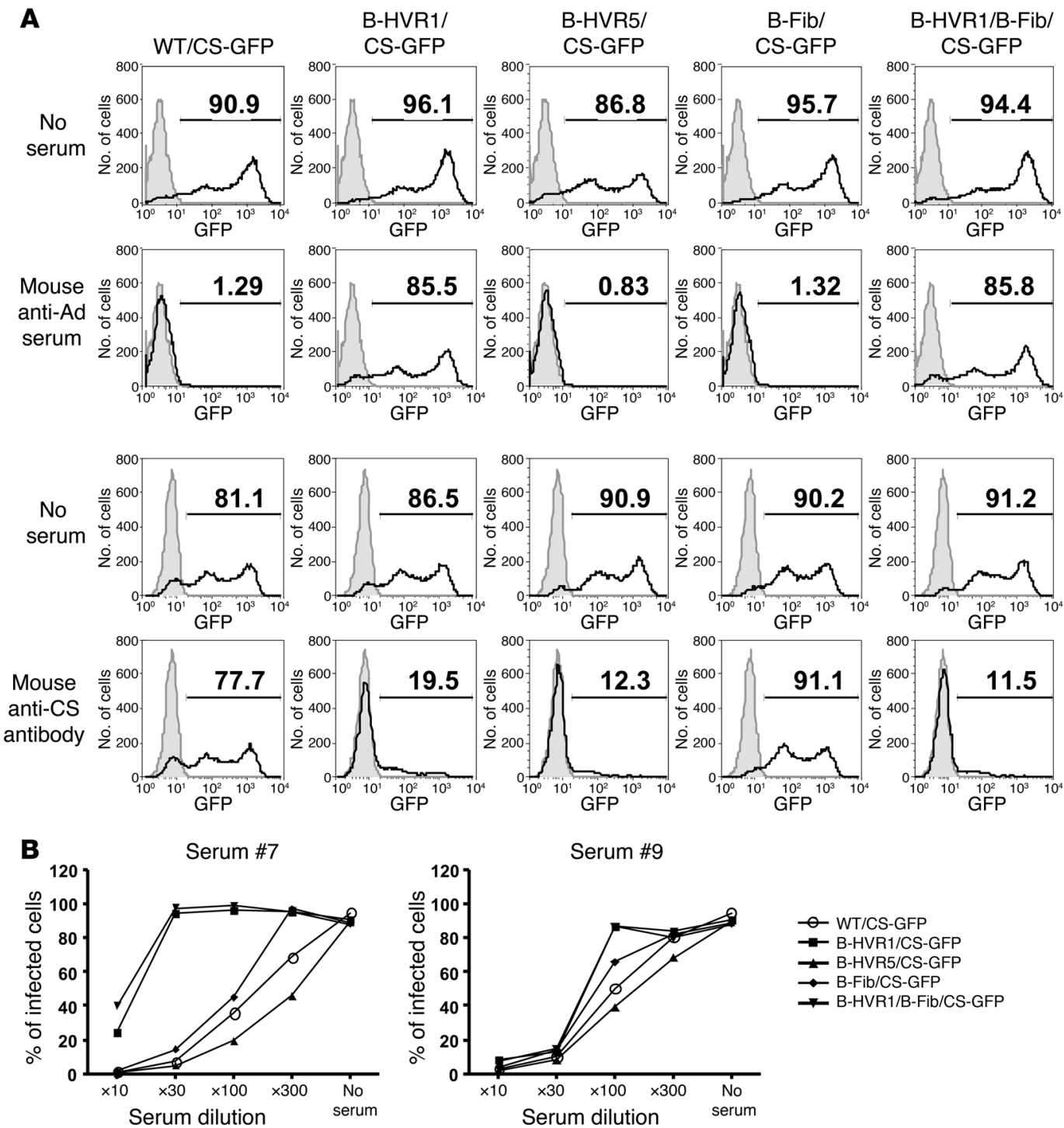

Serum \#14

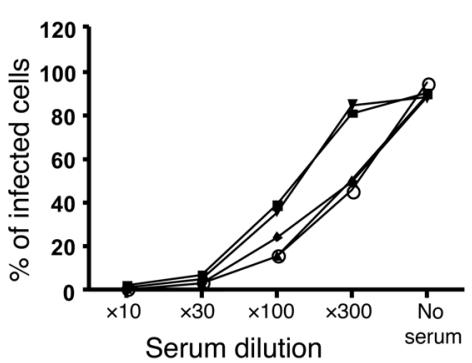

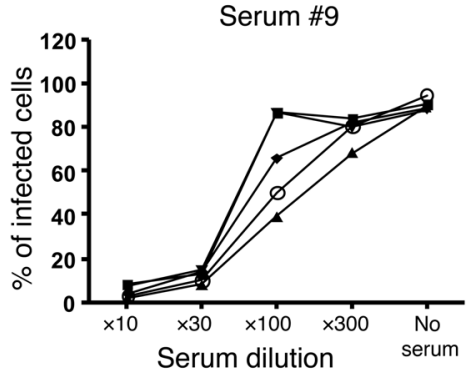

Serum \#15

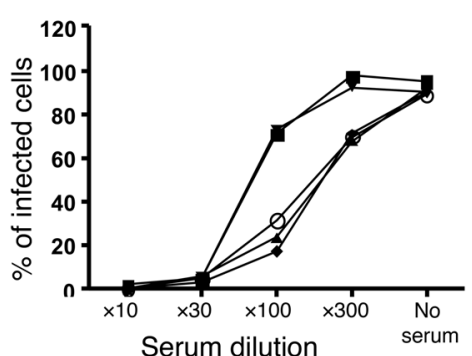

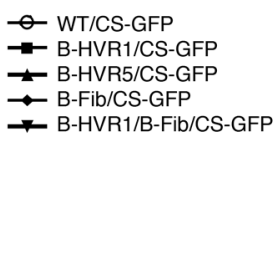

Serum \#16

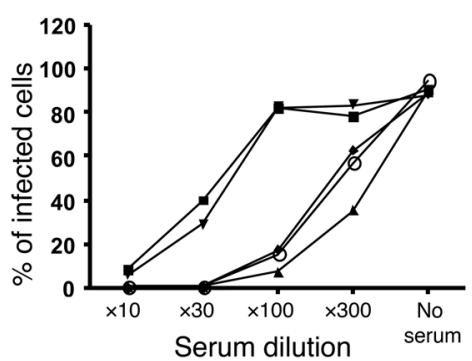

Figure 9

In vitro neutralization of capsid-modified rAd with human and mouse anti-Ad sera as well as anti-P. yoelii CS antibody. (A) In vitro neutralization of capsid-modified rAd by mouse anti-Ad serum and anti- $P$. yoelii CS antibody. AD293 cells were infected with WT/GFP at a ratio of 1:250 (cell/Ad particle) in the presence of normal BALB/c serum, mouse anti-Ad serum (300-fold dilution), or purified mouse polyclonal anti- $P$. yoelii CS antibody $(3 \mu \mathrm{g} / \mathrm{ml})$. After 24-hour incubation, cells were analyzed by flow cytometry to measure GFP expression. Filled areas represent cells without rAd, and solid lines represent cells with rAd. The numbers indicate the percentage of cells that stain positive by a flow cytometric analysis. (B) In vitro neutralization of capsid-modified rAd by Ad-immune human sera. AD293 cells were infected with the rAd in the presence of human sera at the indicated dilutions. Data are shown as the percentages of infected cells (GFP-positive cells measured by flow cytometry) at the indicated serum dilution. 

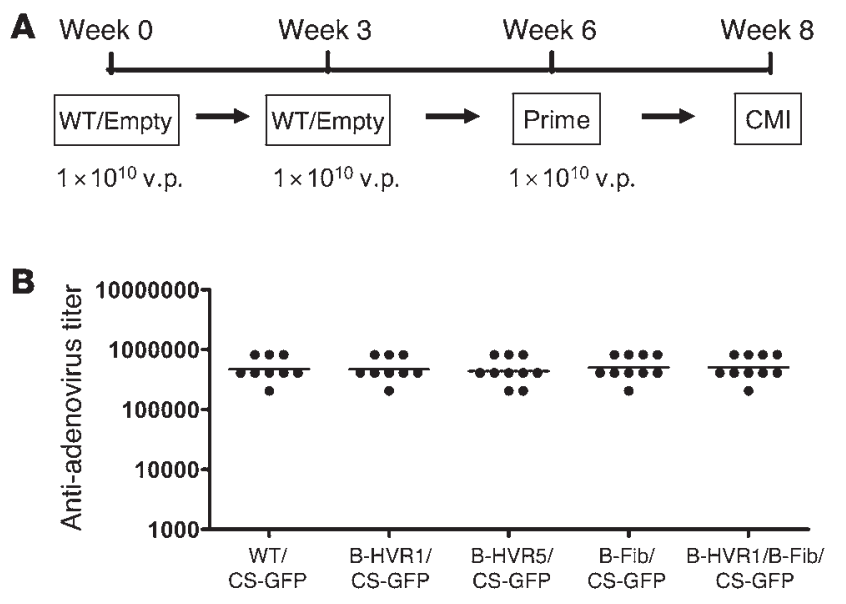
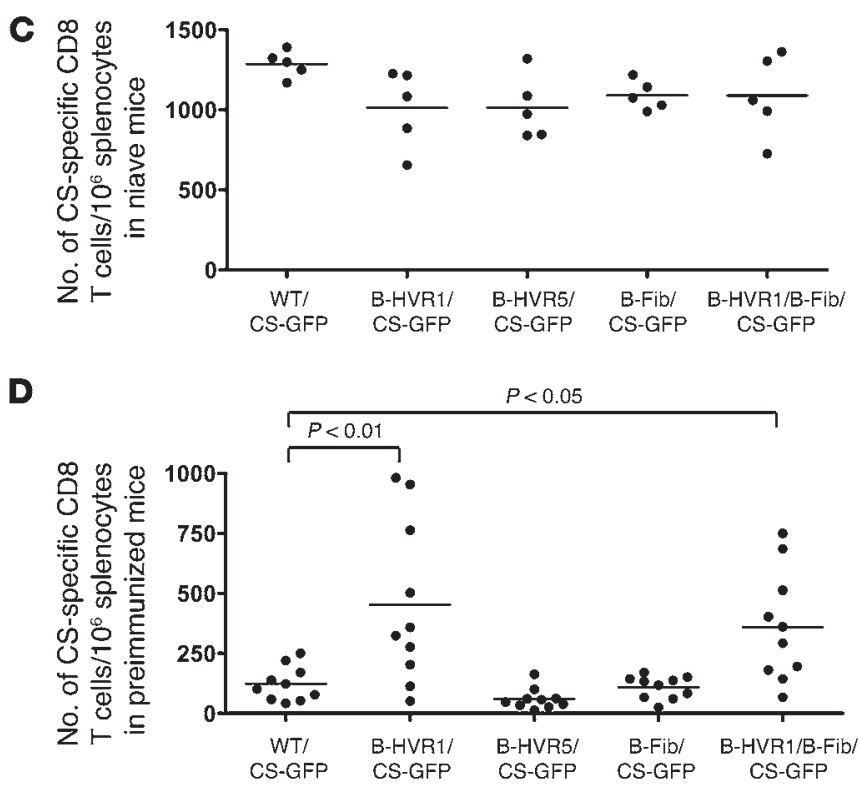

\section{Figure 10}

Effect of preexisting anti-Ad immunity on the induction of $P$. yoelii CS-specific CD8 ${ }^{+}$T cell response by capsid-modified rAd. (A) Immunization regimen. BALB/c mice were immunized intramuscularly with $10^{10} \mathrm{v} . \mathrm{p}$. WT/Empty at weeks 0 and 3 , and then randomized into 5 groups (10 mice in each group) based on anti-Ad titers at week 6 . The randomized mice and naive mice were immunized with $10^{10} \mathrm{~V} . \mathrm{p}$. of capsid-modified rAd at week 6. $P$. yoelii CS-specific CD8 $8^{+} \mathrm{T}$ cell responses were measured by IFN- $\gamma$ ELISPOT assay at week 8. (B) Anti-Ad antibody titer after randomization at week 6 . Antibody titers were determined by ELISA, using plates coated with WT/Empty Ad. (C) $P$. yoelii CS-specific CD8+ T cell response in mice without preimmunization with WT/Empty. Data are shown as the number of IFN- $\gamma-$ secreting $P$. yoelii CS-specific T cells in 1 million splenocytes. (D) $P$. yoelii CS-specific CD8 $8^{+}$T cell response in mice preimmunized with WT/Empty at weeks 0 and 3 . One-way ANOVA, followed by a Dunnett's test, was used to determine the differences between the group immunized with WT/CS-GFP and each of the other groups immunized with capsid-modified rAd. Horizontal bars represent the mean.

(5'-GGACAGGGCCCTGGAGCTCCACAGGGACCAGGTGCACCTCAAGGGCCTGGAGCCCCTGACACAACTCCAAGTGCATAC-3' and 5'-GTCAGGGGCTCCAGGCCCTTGAGGTGCACCTGGTCCCTGTGGAGCTCCAGGGCCCTGTCCTGTTTCCTGTGTACCGT-3'). The PCR product was digested with EcoNI and $\mathrm{MFeI}$ and then used to replace the native EcoNI-MfeI region of fiber in pUC19 vector. After confirming the sequence, the SpeI-PacI fragment of AdEasy-1 was replaced with the SpeIPacI fragment containing the epitope sequence.

For HVR1 modification, AdEasy-1 was digested with Sfil, and the 6.4-kbp fragment was subcloned into EcoRI and PstI sites of pUC19, using EcoRISfiI and PstI-SfiI linker oligomers. To replace HVR1 with a PyCS-B cell epitope, the region containing AgeI and NdeI sites was amplified by 2 -step PCR, using primers that have the epitope instead of HVR1 sequence (5'CACAGGGACCAGGTGCACCTCAAGGGCCTGGAGCCCCTAAAACTCACGTATTTGGGCAGGC-3' and 5'-TGAGGTGCACCTGGTCCCTGTGGAGCTCCAGGGCCCTGTTCATCCCATTCGCAAGGATTTG-3'). The PCR product was digested with AgeI and NdeI, and then used to replace the native AgeI-NdeI region of SfiI fragment in pUC19 vector. After confirming the sequence, the SfiI fragment of AdEasy-1 was replaced with the SfiI fragment containing the epitope sequence. For HVR5 modification, the XbaI site was introduced into HVR5 in L1 loop of the hexon in AdEasy-1, and then a synthesized, phosphorylated double-stranded oligomer coding the epitope was inserted into the XbaI site (15). The insertion was confirmed by sequencing. WT/Empty genome was constructed using the original pshuttle-CMV vector for homologous recombination.

For construction of P. falciparum CS protein (PfCSP) Ad, the PfCSP coding sequence, except for the GPI-anchored sequence, was codon-optimized and synthesized by Integrated DNA Technologies Inc. The opti- mized PfCSP fragment was inserted between KpnI and HindIII sites of pShuttle-CMV to make WT/PfCSP Ad. HVR1 of WT/PfCSP was modified by inserting (NANP) 4 sequence as described above to construct NANPHVR1/PfCSP. All primers and linker oligomers were synthesized by Integrated DNA Technologies Inc.

Purified rAd genome was digested with PacI and used for transfection of AD293 cells to produce rAd. After Ad amplification, Ad was purified by $\mathrm{CsCl}$ gradient centrifugation. v.p. was calculated based on $\mathrm{OD}_{260}$ $\left(1 \mathrm{OD}_{260}=1.25 \times 10^{12} \mathrm{v} \cdot \mathrm{p} . / \mathrm{ml}\right)$.

Silver staining and Western blot analysis. Virions $\left(2 \times 10^{9}\right.$ v.p./lane for silver staining and $1 \times 10^{10} \mathrm{v} . \mathrm{p}$./lane for Western blot analysis) were lysed in SDS sample buffer and applied onto a $4 \%-12 \%$ gradient polyacrylamide gel under a reducing condition. After electrophoresis, the gel was stained with a silver staining kit (Invitrogen). For Western blotting of capsid-modified rAd, the separated proteins were blotted onto a nitrocellulose membrane. The membrane was blocked in 3\% skim milk and then incubated with Protein G-purified mouse polyclonal anti-P. yoelii sporozoite antibody. After washing, the blots were incubated with HRP-labeled goat anti-mouse IgG antibody (Thermo Fisher Scientific Inc.), and the signal was developed using an ECL detection system (Invitrogen).

To detect $P$. yoelii CS protein expression after infection with capsidmodified rAd, $5 \times 10^{5}$ HeLa cells were infected with $5 \times 10^{9}$ v.p. of capsidmodified rAd. Whole cell lysates were prepared 24 hours after infection. After SDS-PAGE electrophoresis and blotting to a nitrocellulose membrane, P. yoelii CS protein was detected with mouse monoclonal antiP. yoelii CS antibody (9D3).

Ad ELISA assay. The rAds were serially diluted in $0.1 \mathrm{M}$ sodium carbonate buffer ( $\mathrm{pH}$ 9.5) and immobilized on Maxisorp ELISA plates (Thermo Fisher 

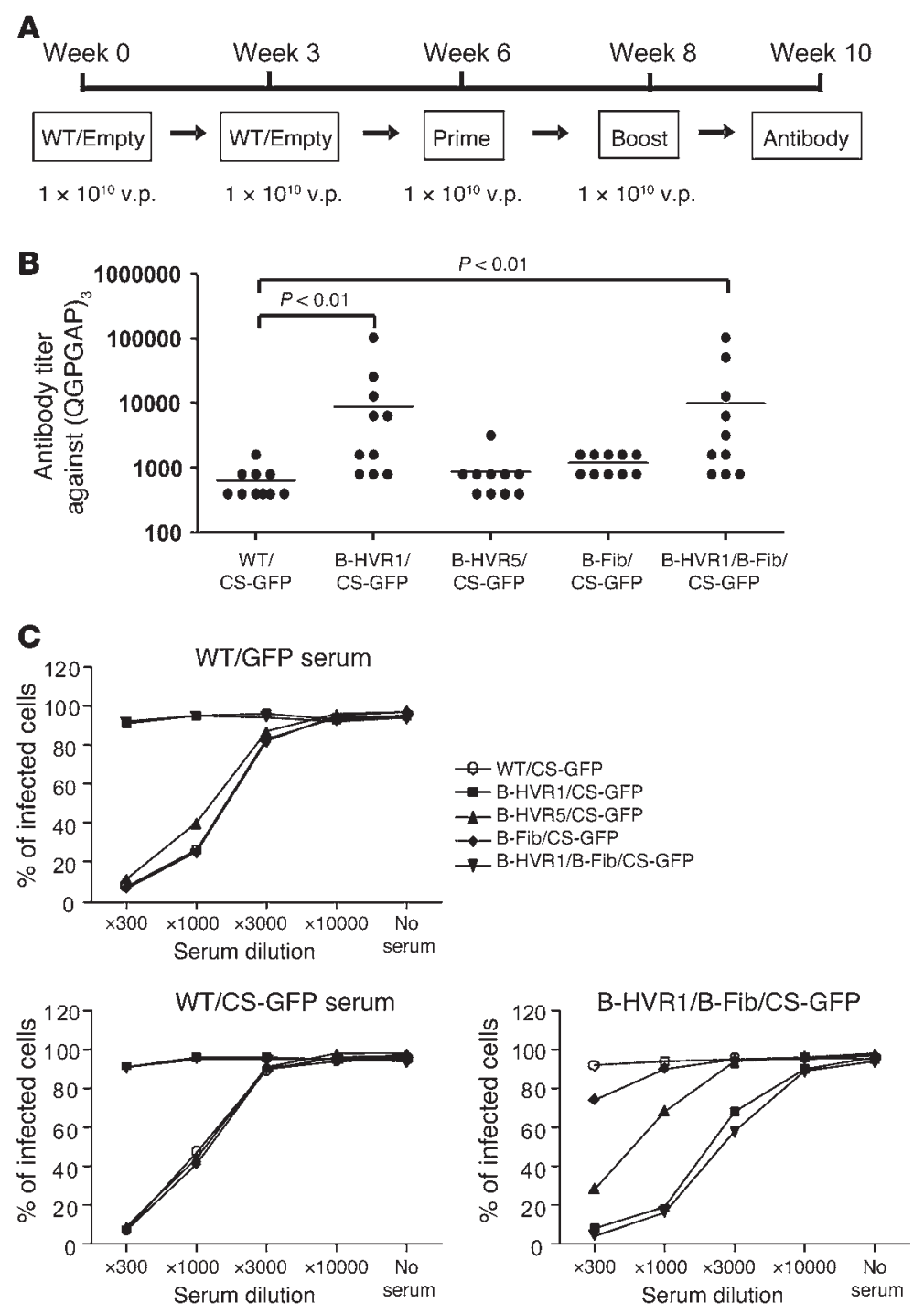

Figure 11

Effect of preexisting anti-Ad immunity on the induction of anti-(QGPGAP) ${ }_{3}$ antibody response by capsid-modified rAds and on their in vitro infectivity. (A) Immunization regimen. BALB/c mice were first immunized intramuscularly with $10^{10}$ v.p. WT/Empty at weeks 0 and 3, followed by immunization of $10^{10}$ v.p. capsid-modified rAd at weeks 6 and 8 . Titers of anti-(QGPGAP) $)_{3}$ antibody were measured by ELISA at week 10. (B) Anti-(QGPGAP) $)_{3}$ antibody titers in rAd-immunized mice preimmunized with WT/Empty at weeks 0 and 3. One-way ANOVA, followed by a Dunnett's test, was used to determine the differences between the group immunized with WT/CSGFP and each of the other groups immunized with capsid-modified rAd. Horizontal bars represent the geometric mean. (C) In vitro neutralization of capsid-modified rAd by serum from mice immunized with various rAds. Mouse sera were prepared from BALB/c mice (10 mice in each group) immunized twice with $1 \times 10^{9}$ v.p. WT/ GFP, WT/CS-GFP, or B-HVR1/B-Fib/CS-GFP. These sera were pooled in each group, and neutralizing activity was evaluated as described in Figure 9.
Scientific Inc.) at $4{ }^{\circ} \mathrm{C}$ overnight. After extensive washes with PBST $(0.05 \%$ Tween-20 in PBS), the plates were blocked with $1 x$ Diluent (eBioscience) for 1 hour at room temperature and incubated with mouse anti-recombinant $P$. yoelii CS protein polyclonal antibody for 1 hour at room temperature. The bound antibody was detected with HRP-labeled goat anti-mouse IgG antibody. After washing, 3,3',5,5'-tetramethylbenzidine was added to the plates, and the reaction was stopped by adding $2 \mathrm{~N} \mathrm{H}_{2} \mathrm{SO}_{4}$. The OD was measured at $450 \mathrm{~nm}$.

Polyclonal anti-recombinant $P$. yoelii CS protein antibody was purified with Protein $G$ column from BALB/c mouse serum immunized with recombinant $P$. yoelii CS protein in combination with incomplete Freund's adjuvant 3 times. From 31 to 405 aa of $P$. yoelii CS protein (GenBank AAA29528.1), with a 6xHis-Tag at the C terminus, was expressed in Pichia pastoris using PPIC9K expression vector (Invitrogen), and recombinant P. yoelii CS protein was purified from culture supernatant with $\mathrm{Ni}^{+} \mathrm{NTA}$ column (Qiagen) in a native condition.

Ad infectious unit titration. Infectious units of Ad were determined using end point dilution assay. $2 \times 10^{4} \mathrm{AD} 293$ cells were seeded in 96-well plates, and then 10 -fold serial dilutions of Ad were added to the plates (12 wells for 1 dilution). The plates were incubated for 7 days at $37^{\circ} \mathrm{C}, 5 \% \mathrm{CO}_{2}$, and then the number of wells that had GFP-positive cells was counted. Infectious units were calculated based on the following equation: infectious units per $\mathrm{ml}=10^{(X+0.8)}$, where $X$ is the sum of the fractions of GFP-positive wells in each dilution.

To evaluate Ad productivity, AD293 cells were infected with $2 \times 10^{7}$ capsid-modified rAd in triplicate wells and incubated at $37^{\circ} \mathrm{C}, 5 \% \mathrm{CO}_{2}$, for 2 days after infection. Crude Ad solution was prepared from the infected cells by 5 cycles of freeze-thaw. Infectious units of the crude Ad solutions were determined by the end point dilution method described above.

In vitro Ad infection assay. Mouse CAR cDNA clone (I.M.A.G.E. clone ID 4216727) was obtained from ATCC. A CAR expression plasmid was constructed by amplifying the coding sequence with primers (5'-CTAGAAGCTTACCTGCAAGCCACGACC-3' and 5'-GTCAGGGCCCTATACCACTGTAATGCCATCG-3') that have HindIII and ApaI sites, respectively, and then cloned into the same sites of pcDNA3.1 (+) (Invitrogen). After confirming the sequence, the plasmid was used for stable transfection using the Nucleofector Kit (Lonza) to establish stable clones. The transfected cells were cloned by limiting dilution in the presence of $1.2 \mathrm{mg} / \mathrm{ml}$ (A20) or $1 \mathrm{mg} / \mathrm{ml}$ (L1210) of Geneticin (Invitrogen), and then clones that showed higher susceptibility to WT/GFP infection were screened. 
Immature mouse bone marrow DCs were prepared by culturing bone marrow progenitor cells from BALB/c mice with $10 \mathrm{ng} / \mathrm{ml}$ mouse GM-CSF (BD Biosciences) for 7 days. For an in vitro Ad infection assay, AD293 cells, mouse DCs, mouse CAR/A20 cells, or mouse CAR/L1210 cells were seeded in a 48 -well plate $\left(2 \times 10^{5}\right.$ cells/well), and then capsid-modified or unmodified Ad was added at a cell to Ad particle ratio of 1:250, 1:50,000, 1:1,000, or 1:600 to each group of cells, respectively. After 24-hour incubation, infected cells were analyzed with FACS Calibur System (BD Biosciences) to measure GFP expression.

For the in vitro Ad neutralization experiments, serum or antibody was added to the cells at the indicated dilutions prior to the Ad infection. Eighteen human serum samples ( 9 males of European descent, 20 to 45 years old; 9 females of European descent, 20 to 47 years old) were obtained from Innovative Research, and their neutralizing activity was evaluated as described above. All flow cytometry data was analyzed with FlowJo version 8.8 software (Tree Star Inc.).

Immunizations. Six- to 8-week-old female BALB/c mice were purchased from Taconic and maintained under standard conditions in the Laboratory Animal Research Center of The Rockefeller University. For immunization, Ads were diluted in PBS and injected intramuscularly at indicated doses. All animal studies were approved by the Institutional Animal Care and Use Committee at The Rockefeller University.

Assessment of CS protein-specific cellular and humoral responses. The numbers of P. yoelii CS-specific, IFN- $\gamma$-secreting $\mathrm{CD}^{+} \mathrm{T}$ cells in the spleens of immunized mice were determined by an ELISPOT assay, using a synthetic peptide corresponding to the $\mathrm{CD}^{+} \mathrm{T}$ cell epitope (SYVPSAEQI) within the P. yoelii CS protein, as previously described (17). P. yoelii CS-specific humoral response was determined by ELISA. Maxisorp ELISA plates were coated with $5 \mu \mathrm{g} / \mathrm{ml}$ P. yoelii CS-specific peptide (QGPGAP) ${ }_{3}$ or (PPQQ) 4 in $0.1 \mathrm{M}$ sodium carbonate buffer ( $\mathrm{pH} 9.5$ ) at $4^{\circ} \mathrm{C}$ overnight. After blocked with $1 \mathrm{x}$ Diluent, the plates were washed again and $100 \mu \mathrm{l}$ of serially 2 -fold-diluted plasma or serum in $1 x$ Diluent was added to the plates. After 1-hour incubation, the plates were washed and incubated with 100 $\mu$ l of HRP-labeled goat anti-mouse IgG antibody. All of the peptides were synthesized by Biosynthesis.

IFA was done to determine anti-sporozoite antibody titers (17). Briefly, air-dried sporozoites on multi-sport glass slides were incubated with $3 \%$ BSA in PBS for 1 hour and then incubated with diluted plasma for 1 hour in a humidified chamber at room temperature. After washing, the slides were incubated with Alexa Fluor 488-labeled goat anti-mouse antibody (Invitrogen) for 1 hour. After washing, the slides were mounted with mounting medium (KPL), and IFA titers were determined as the highest dilution producing fluorescence under a fluorescent microscope.

In order to measure PfCSP-specific humoral immune response, ELISA assay was done as described above, except that ELISA plates were coated with $1 \mu \mathrm{g} / \mathrm{ml}(\mathrm{T} 1 \mathrm{~B})_{4}$ peptide that contains NANP repeat sequence (31). $(\mathrm{T} 1 \mathrm{~B})_{4}$ peptide was provided by Elizabeth Nardin, New York University School of Medicine, New York, New York, USA.

Sporozoite challenge and assessment of parasite burden in the liver and parasitemia in the blood. P. yoelii (17XNL strain) sporozoites were obtained from dissected salivary glands of infected Anopheles stephensi mosquitoes 2 weeks after infective blood meal. Sporozoite challenge experiment was done as described previously (17). Briefly, immunized mice were injected with $2 \times 10^{4}$ live P. yoelii sporozoites (provided by Ana Rodriguez, New York University School of Medicine) via tail vain, and 42 hours after the challenge - when the parasite fully matures in the hepatocyte - parasite burden in the liver was determined by measuring parasite-specific ribosomal RNA using 7300 Real-Time PCR System (Applied Biosystems). Parasite burden was described as a ratio of the absolute copy number of parasite ribosomal RNA to that of mouse GAPDH mRNA.
For evaluation of protection against blood-stage malaria infection, $\mathrm{BALB} / \mathrm{c}$ mice were immunized at weeks 0,3 , and 6 at doses of $10^{8}, 10^{9}$, and $10^{10} \mathrm{v} . p$. , respectively, and challenged with 50 sporozoites intravenously at week 10. Giemsa-stained blood smears were analyzed 3-12 days after challenge to detect blood-stage malaria parasite infection.

In vitro sporozoite neutralization assay. Human CD81 cDNA (I.M.A.G.E. clone ID 3510634) was obtained from ATCC, and the coding region was PCR amplified and cloned into KpnI and HindIII sites of pcDNA3.1 (-) (Invitrogen). The human CD81-expressing HepG2 cell line was established by transfecting CD81/pcDNA3.1 (-), and cloning was performed by limiting dilution in the presence of $0.8 \mathrm{mg} / \mathrm{ml} \mathrm{Geneticin} \mathrm{(Invitrogen).} \mathrm{CD81-}$ expressing clones were selected by flow cytometry analysis, using APClabeled anti-human CD81 antibody (BD Biosciences).

For a sporozoite neutralization assay, CD81/HepG2 cells were seeded onto a 96-well plate at $2 \times 10^{5}$ cells per well and incubated for overnight at $37^{\circ} \mathrm{C}, 5 \% \mathrm{CO}_{2}$. P. yoelii sporozoites, prepared as described above, were purified using DE52 anion exchange column (Whatman) to reduce bacterial contamination (32), and then, $5 \times 10^{4}$ sporozoites per well were added to the CD81/HepG2 cells in the presence of mouse serum. After 2-hour incubation, uninfected sporozoites were washed out with medium, and then the cells were cultured for 42 hours. Total RNA was prepared using TRIzOL (Invitrogen), and the relative amount of parasite ribosomal RNA to human GAPDH was measured by real-time PCR.

In vivo Ad neutralization. Mice were immunized intramuscularly with WT/Empty at a dose of $10^{10}$ v.p. at weeks 0 and 3 to induce preexisting immunity to WT Ad5. One day before week 6, sera were collected from immunized mice, and anti-Ad titers were determined by an ELISA using Ad particle-coated plates. The mice were randomized based on anti-Ad titers and then immunized with capsid-modified Ad at a dose of $10^{10} \mathrm{v} \cdot \mathrm{p}$. at week 6. Two weeks after capsid-modified Ad injection, P. yoelii CS-specific $\mathrm{CD}^{+} \mathrm{T}$ cell response was measured by an IFN- $\gamma$ ELISPOT assay, as described above.

Statistics. All of the statistical analyses were done using GraphPad Prism (version 4.03) (GraphPad Software Inc.). Horizontal bars in each figure represent the geometric mean (antibody titer and protection) or mean (ELISPOT). In the protection experiment in which the parasite load in the liver was determined by a real-time RT-PCR, the values were log transformed, and then 1-way ANOVA, followed by a Dunnett's test, was used to determine the differences between the group immunized with WT/CS-GFP and each of the other groups immunized with capsid-modified CS-GFP Ad. For the experiment in which a sterile immunity was assessed, Fisher's exact test was applied. For a correlation analysis, linear regression analysis was applied to log-transformed anti$(\mathrm{QGPGAP})_{3}$ antibody titers and the log-transformed ratio of parasite ribosomal RNA to mouse GAPDH.

\section{Acknowledgments}

We thank the MR4 Resource Center at ATCC and Ana Rodriguez for providing P. yoelii sporozoites and Vincent Sahi for FACS analysis. This work was supported by a grant from the NIH (R01 AI081510 to M. Tsuji), the Bill and Melinda Gates Foundation, the Irene Diamond Foundation, and Otsuka Pharmaceutical Co. Ltd.

Received for publication May 14, 2010, and accepted in revised form July 14, 2010.

Address correspondence to: Moriya Tsuji, HIV and Malaria Vaccine Program, Aaron Diamond AIDS Research Center, 455 First Avenue, New York, New York 10016, USA. Phone: 212.448.5021; Fax: 212.725.1126; E-mail: mtsuji@adarc.org. 
1. World Health Organization. World Malaria Report 2005. http://rbm.who.int/wmr2005/. Accessed May 13, 2010.

2. Nussenzweig RS, Vanderberg J, Most H, Orton C. Protective immunity produced by the injection of $\mathrm{x}$-irradiated sporozoites of plasmodium berghei. Nature. 1967;216(5111):160-162.

3. Gwadz R, Cochrane AH, Nussenzweig V, Nussenzweig RS. Preliminary studies on vaccination of rhesus monkeys with irradiated sporozoites of Plasmodium knowlesi and characterization of surface antigens of these parasites. Bull World Health Organ. 1979;57 suppl 1:165-173.

4. Clyde DF, Most H, McCarth VC, Vanderberg JP. Immunization of man against sporozite-induced falciparum malaria. Am J Med Sci. 1973;266(3):169-177.

5. Edelman R, et al. Long-term persistence of sterile immunity in a volunteer immunized with $\mathrm{X}$-irradiated Plasmodium falciparum sporozoites. J Infect Dis. 1993;168(4):1066-1070.

6. Nussenzweig V, Nussenzweig RS. Circumsporozoite proteins of malaria parasites. Cell. 1985 42(2):401-403.

7. Nardin EH, et al. Circumsporozoite proteins of human malaria parasites Plasmodium falciparum and Plasmodium vivax. J Exp Med. 1982;156(1):20-30.

8. Tsuji M, Zavala F. T cells as mediators of protective immunity against liver stages of Plasmodium. Trends Parasitol. 2003;19(2):88-93.

9. Rodrigues EG, Zavala F, Eichinger D, Wilson JM, Tsuji M. Single immunizing dose of recombinant adenovirus efficiently induces CD8+ T cell-mediated protective immunity against malaria. J Immunol. 1997;158(3):1268-1274.

10. Anderson RJ, et al. Enhanced CD8+ T cell immune responses and protection elicited against Plasmodium berghei malaria by prime boost immunization regimens using a novel attenuated fowlpox virus. J Immunol. 2004;172(5):3094-3100.

11. Bruña-Romero O, González-Aseguinolaza G, Hafalla JC, Tsuji M, Nussenzweig RS. Complete, long-lasting protection against malaria of mice primed and boosted with two distinct viral vectors expressing the same plasmodial antigen. Proc Natl Acad Sci U S A. 2001;98(20):11491-11496.

12. Tao D, Barba-Spaeth G, Rai U, Nussenzweig V, Rice CM, Nussenzweig RS. Yellow fever $17 \mathrm{D}$ as a vaccine vector for microbial CTL epitopes: protection in a rodent malaria model. J Exp Med. 2005; 201(2):201-209.

13. Worgall $S$, et al. Protection against $P$. aeruginosa with an adenovirus vector containing an OprF epitope in the capsid. J Clin Invest. 2005;115(5):1281-1289.

14. McConnell MJ, Danthinne X, Imperiale MJ. Characterization of a permissive epitope insertion site in adenovirus hexon. J Virol. 2006;80(11):5361-5370.

15. Krause A, et al. Epitopes expressed in different adenovirus capsid proteins induce different levels of epitope-specific immunity. J Virol. 2006; 80(11):5523-5530.

16. Worgall S, Krause A, Qiu J, Joh J, Hackett NR, Crystal RG. Protective immunity to pseudomonas aeruginosa induced with a capsid-modified adenovirus expressing P. aeruginosa OprF. J Virol. 2007; 81(24):13801-13808.

17. Ophorst OJ, et al. Immunogenicity and protection of a recombinant human adenovirus serotype 35 based malaria vaccine against Plasmodium yoelii in mice. Infect Immun. 2006;74(1):313-320.

18. Abbink P, et al. Comparative seroprevalence and immunogenicity of six rare serotype recombinant adenovirus vaccine vectors from subgroups $\mathrm{B}$ and $\mathrm{D}$. J Virol. 2007;81(9):4654-4663.

19. Wu H, Dmitriev I, Kashentseva E, Seki T, Wang M, Curiel DT. Construction and characterization of adenovirus serotype 5 packaged by serotype 3 hexon. J Virol. 2002;76(24):12775-12782.

20. Roberts DM, et al. Hexon-chimaeric adenovirus serotype 5 vectors circumvent pre-existing anti-vector immunity. Nature. 2006;441(7090):239-243.

21. Bergelson JM, et al. Isolation of a common receptor for Coxsackie B viruses and adenoviruses 2 and 5 .
Science. 1997;275(5304):1320-1323.

22. Silvie O, et al. Expression of human CD81 differently affects host cell susceptibility to malaria sporozoites depending on the Plasmodium species. Cell Microbiol. 2006;8(7):1134-1146.

23. Tatsis N, Ertl HC. Adenoviruses as vaccine vectors. Mol Ther. 2004;10(4):616-629.

24. Kalyuzhniy O, et al. Adenovirus serotype 5 hexon is critical for virus infection of hepatocytes in vivo. Proc Natl Acad Sci U S A. 2008;105(14):5483-5488.

25. Hsu C, Boysen M, Gritton LD, Frosst PD, Nemerow GR, Von Seggern DJ. In vitro dendritic cell infection by pseudotyped adenoviral vectors does not correlate with their in vivo immunogenicity. Virology. 2005;332(1):1-7.

26. Charoenvit Y, et al. Monoclonal, but not polyclonal, antibodies protect against Plasmodium yoelii sporozoites. J Immunol. 1991;146(3):1020-1025.

27 . Wang $\mathrm{R}$, et al. Induction of protective polyclonal antibodies by immunization with a Plasmodium yoelii circumsporozoite protein multiple antigen peptide vaccine. J Immunol. 1995;154(6):2784-2793.

28. Pichla-Gollon SL, et al. Structure-based identification of a major neutralizing site in an adenovirus hexon. J Virol. 2007;81(4):1680-1689.

29. Abe $\mathrm{S}$, et al. Adenovirus type 5 with modified hexons induces robust transgene-specific immune responses in mice with pre-existing immunity against adenovirus type 5. J Gene Med. 2009;11(7):570-579.

30. Grote A, et al. JCat: a novel tool to adapt codon usage of a target gene to its potential expression host. Nucleic Acids Res. 2005;33(Web Server issue):W526-W531.

31. Calvo-Calle JM, Oliveira GA, Watta CO, Soverow J, Parra-Lopez C, Nardin EH. A linear peptide containing minimal T- and B-cell epitopes of Plasmodium falciparum circumsporozoite protein elicits protection against transgenic sporozoite challenge. Infect Immun. 2006;74(12):6929-6939.

32. Mack SR, Vanderberg JP, Nawrot R. Column separation of Plasmodium berghei sporozoites. J Parasitol. 1978;64(1):166-168. 